\title{
A Theory of Quantifiable Beliefs
}

\author{
Simon Grant \\ School of Economics \\ Faculty of Economics and Commerce \\ Australian National University \\ Email: Simon.Grant@anu.edu.au \\ Edi Karni \\ Department of Economics \\ John Hopkins University \\ Email: karni@jhunix.hcf.jhu.edu
}

\begin{abstract}
November 2000
Working Papers in Economics and Econometrics No. 388

Australian National University
\end{abstract}

ISBN: 0868313885 
November, 2000

\title{
A Theory of Quantifiable Beliefs*
}

\begin{abstract}
Building upon the works of Anscombe and Aumann (1963) and Karni and Schmeidler (1981), we develop a general axiomatic theory of quantifiable beliefs - a form of probabilistic sophistication that does not preclude state-dependent preferences and does not require the reduction of compound lotteries. The theory includes the state-dependent expected utility model of Karni and Schmeidler (1981) and the state-independent non-expected utility model of Machina and Schmeidler (1995) as special cases. The theory is flexible enough to admit recursivity in the decision-making process. One specific example of this recursive class is shown to be compatible with a quantifiable beliefs version of Schmeidler's (1989) Choquet expected utility maximizing model and thus capable of rationalizing Ellsberg-paradox type behavior.

Keywords: subjective probability, quantifiable beliefs, state-dependent preferences, probabilistic sophistication, non-expected utility.
\end{abstract}

JEL classifications: D80, D81

\author{
Simon Grant \\ Department of Economics \\ Faculty of Economics and Commerce \\ Australian National University \\ email: Simon.Grant@anu.edu.au \\ Edi Karni \\ Department of Economics \\ Johns Hopkins University \\ email: karni@jhunix.hcf.jhu.edu
}

\footnotetext{
*This research was undertaken during Grant's visit to Johns Hopkins University in Spring 2000. He gratefully acknowledges the hospitality and financial support provided by Johns Hopkins. We benefited from comments of Mark Machina and David Kelsey and from Mordecai Kurz's insistence on a better expalnation of concordance.
} 


\section{Introduction}

In subjective expected utility theory (henceforth SEU) choice under uncertainty is perceived as the maximization of the mathematical expectation of the utility function with respect to the subjective probabilities (see Savage [1954] for the definitive statement and Anscombe and Aumann [1963] for an alternative, simpler, treatment.) Presumably individual choicebehavior is the culmination of two distinct, if implicit, cognitive processes: The assessment of the likely realization of alternative events, or the formation of beliefs, and the evaluation of the consequences. Moreover, the decision-maker's beliefs are representable by (subjective) probabilities and his evaluation of consequences is representable by a utility function.

Recently Machina and Schmeidler (1992, 1995) argued that the issue of quantifying decision-makers' beliefs by a probability measure is separate from the other tenets of SEU. ${ }^{1}$ In other words, the notion that decision-makers' beliefs may be represented by probability measures does not depend on their being expected utility maximizers. Machina and Schmeidler (henceforth MS) introduce the term probabilistically sophisticated choice to describe the behavior of a decision-maker who "...assigns subjective probabilities to events and judges each act solely on the basis of its implied probability distribution over outcomes, but does not necessarily rank these probability distributions according to the expected utility principle." (Machina and Schmeidler [1995, p. 109].) As in SEU theory, probabilistic sophistication, since it requires that acts be judged "solely on the basis of its implied probability distribution over outcomes," precludes circumstances in which the implicit evaluation of consequences is not independent from the underlying events in which they arise. For example, probabilistic sophistication theory does not apply to the choice of disability insurance or life insurance policies since, in these instances, the evaluation of the monetary payoff is, in general, not independent of the disability or whether the individual is dead or alive. ${ }^{2}$ Yet, there is no reason to suppose that in situations involving "state-dependent evaluation of consequences" decision-makers do not form beliefs about the likely realization of events, or that these beliefs are qualitatively different from beliefs in the case of state-independent preferences and may not be represented by probabilities.

Another implicit aspect of the SEU models that is shared by probabilistic sophistication theory, is the reduction of compound lotteries. By insisting that choice problems amount to comparisons of probability distributions over outcomes SEU and probabilistically sophistication disregard the possibility that decision-makers assess relative likelihoods of events

\footnotetext{
1 An extension and further elaboration of the Machina-Schmeidler model is contained in Epstein and LeBreton (1993) and Grant (1995).

${ }^{2}$ For additional examples and more detailed discussion of state-dependent preferences see Karni (1985).
} 
in terms of probabilities and yet, when facing compound uncertainty, do not abide by the reduction principle. In particular, probabilistic sophistication is incompatible with probabilistically sophisticated Choquet expected utility maximizing behavior (e.g., a probability sophisticated version of Schmeidler's [1989] model). ${ }^{3}$

In the present paper we introduce the concept of quantifiable beliefs - a form of probabilistic sophistication that does not preclude state-dependent preferences and does not require the reduction of compound lotteries. We develop a general axiomatic theory of quantifiable beliefs that includes the models of Machina and Schmeidler (1995) and Karni and Schmeidler (1981) as special cases. To underscore the difference between the theory of quantifiable beliefs and the MS theory of probabilistically sophisticated choice we note a market phenomenon described in Epstein (2000) which, if observed under constant beliefs, contradicts the MS notion of probabilistically sophisticated choice. ${ }^{4}$ Epstein observes, however, that this phenomenon does not contradict state-dependent subjective expected utility. Consequently, it is not inconsistent with the theory of quantifiable beliefs.

Building upon the works of Anscombe and Aumann (1963) (henceforth AA) and Karni and Schmeidler (1981) (henceforth KS) our analytical framework includes two main components: First, the horse/roulette lotteries representation of alternative courses of action. Thus, as in AA, courses of action, or acts, are functions from a finite set of states of nature to the set of simple lotteries on an arbitrary set of outcomes. Second, a set of hypothetical interim-state lotteries. These should be conceived of as acts in the AA sense with objective probability assignments to the states of nature. This component is similar to the prize-state lotteries of KS. ${ }^{5}$ However, unlike KS where reduction of compound lotteries is presupposed, the interim-state lotteries structure is more flexible and admits recursivity in the decisionmaking process. Finally, as in KS, the two components are assumed to be consistent in the sense that, for some probability distribution on the states, the preference ranking of acts agrees with the ranking of the corresponding interim-state lotteries.

To grasp the meaning and significance of the hypothetical interim-state lotteries it is useful to consider their application in SEU. To begin with, notice that a decision-maker's beliefs may be viewed as having a cognitive meaning independent of the act of choice (i.e., as

\footnotetext{
${ }^{3}$ Obviously, in general SEU theory is incompatible with Choquet expected utility.

${ }^{4}$ More specifically, let $\theta_{s}$ denote the price of an elementary Arrow security that pays $\$ 1$ in state $s$ and $\$ 0$ in every other state. Denote by $q_{s}$ an investor's stock-demand for such a security. Then, for any two states of nature, say $s$ and $t$, the observations: (a) $\theta_{s} \geq \theta_{t}$ and $\theta_{s}^{\prime} \leq \theta_{t}^{\prime}$, with at least one inequality strict, and (b) $q_{s}>q_{t}$ and $q_{s}^{\prime}<q_{t}^{\prime}$, are inconsistent with the MS concept of probabilistically sophisticated choice.

${ }^{5}$ Indeed there exists a natural one-to-one correspondence between the set of interim-state lotteries and the set of prize-state lotteries.
} 
an input in the process of the formation of preferences.) Moreover, beliefs are formalized as a binary relation on a set of events (e.g., subsets of the set of states) that has the interpretation "more likely than." Now, it has been observed (Schervish, Seidenfeld and Kadane [1990], Karni [1996]) that, even supposing that decision-makers act upon their beliefs and that these beliefs are representable by probabilities, it is not necessarily the case that these are the probabilities that the SEU model ascribe to them. This is because in SEU theory the uniqueness of the representation pertains to the joint determination of utility function on consequences and the probability measure over the set of states. The subjective probability measure that figures in the theory of Savage (and in the theory of AA) is jointly determined with a utility function that is the same in every state of nature. However, nothing in the axiomatic structure of these theories implies that the utility functions be state-independent. In other words, the definition of probabilities in SEU theory is based on the convention that the valuation of consequences (and not just lotteries on consequences) is state-independent. ${ }^{6}$

It has recently been noted by Karni and Mongin (2000) that the objective of recovering the decision-maker's true beliefs and their genuine probability representation can be attained in the broader analytical framework of Karni and Schmeidler (1981). In the KS model decision-makers are supposed to be able to express their preferences over prize-state lotteries. Moreover, these preferences satisfy the axioms of expected utility theory and are consistent with the actual preferences over acts. Broadly speaking, the consistency requirement is that the conditional (on the states) preference ranking of lotteries of the actual preferences agree with the conditional preference ranking of state-prize lotteries. The subjective probabilities obtained in this extended framework are the unique representation of decision-makers' beliefs. Karni and Mongin (2000) also note that in the case of state-independent preferences, if the probabilities obtained in the KS model are not the same as those of the AA model then the evaluation of the consequences is not independent of the state in which they are realized and, moreover, the KS definition of subjective probabilities represents the decision-makers true beliefs but the AA does not. ${ }^{7}$

The use of the hypothetical prize-state lotteries and preference relations on such lotteries that do not have choice-behavior empirical counterpart may raise methodological objections. Since we are about to employ an extraneous construct analogous to the prize-state lotter-

\footnotetext{
${ }^{6}$ In fact, SEU requires that the preference ranking of lotteries be state-independent. This is implicit in Savage's theory and is explicit in the theory of AA. However, state-independent preferences in this sense do not imply state-independent utility functions. For a more detailed discussion of this point see Karni (1996).

7 Note that state-independence only requires that the ranking of roulette lotteries be state-invariant. Hence, the utility functions may be state-dependent in the sense of having different intercepts and scales.
} 
ies it is important to address this methodological issue. To start with, Karni and Mongin (2000) argue that in von Neumann and Morgenstern's (1944) expected utility theory the set of consequences is arbitrary. Hence, in principle, this theory is capable of accommodating consequences that are prize-state pairs. Verbal testimony regarding preferences over conceivable prize-state lotteries may provide meaningful information regarding the decision-maker's preferences and beliefs. Furthermore, in so far as their empirical validity is concerned, the $K S$ probabilities are consistent with every aspect of observed choice behavior that is consistent with the AA theory and, in addition, they are consistent with verbal-behavior that may contradict the $A A$ theory. ${ }^{8}$ The same justification applies to the theory presented here.

We assume that decision-makers are capable of expressing preferences between pairs of interim-state lotteries and that these preferences agree, in a sense to be specified, with the decision-maker's actual preferences among acts. Unlike KS we do not require that either the preference relation on interim-state lotteries or the horse/roulette lotteries satisfy the independence axiom of expected utility theory. Hence, our theory accommodates probabilistically sophisticated nonexpected utility choice behavior with state-dependent preferences. The axiomatic structure requires that both the preference relation on interim-state lotteries and the preference relation on acts be continuous weak orders. In addition, the ordinal rankings of outcomes in every state are assumed to be independent of the outcomes in other states, and the preference relations are assumed to monotonic with respect to, appropriately defined, first-order stochastic dominance. Our key assumption, the concordance axiom, establishes a degree of agreement between the preference relation on acts and the corresponding preference relation on interim-state lotteries. Loosely speaking, this agreement requires that if, for some probability distribution over the state-space, the estimates of the conditional probabilities of two states based on preferences among interim-state lotteries is the same as the corresponding estimates based on preferences among acts, then these preferences agree on ranking of all acts that assign the same consequences to all other states.

Section 2 lays out the analytical framework, introduces the axioms and present some preliminary results. Section 3 contains the main representation result. Section 4 contains refinements of our general theory of quantifiable beliefs yielding the models of Anscombe and Aumann (1963), Karni and Schmeidler (1981) and Machina and Schmeidler (1995) as special cases. A further refinement of our model leads to recursive preferences with quantifiable beliefs. One specific example of this final class is shown to be compatible with a quantifiable belief version of Schmeidler's (1989) Choquet expected utility maximizing model and thus capable of rationalizing Ellsberg-paradox type behavior. The proofs of all the results appear

\footnotetext{
${ }^{8}$ See Karni (1996) for a description of such verbal behavior.
} 
in the Appendix.

\section{The Model}

\subsection{Preliminaries}

Let $S=\{1, \ldots, n\}$ be a finite nonempty set. The elements of $S$ are referred to as states of nature. The set of probability measures on $S$ will be denoted by $M$. The degenerate measure $\delta^{s}$ is the element of $M$ that assigns to $s$ the unit probability mass. Subsets of $S$ are events.

For each state $s$ in $S$, let $X_{s}$ be an arbitrary set of prizes that are available if $s$ obtains and denote by $L_{s}$ the set of all simple probability measures or (state) roulette lotteries on $X_{s}$. With slight abuse of notation, for each state $s \in S$ and each prize $x \in X_{s}$ we shall use both $\delta^{x}$ and $x$ to denote the element of $L_{s}$ that assigns to the prize $x$ the unit probability mass.

An act (or horse/roulette lottery) in this context is a mapping from the set of states of nature into the corresponding set of roulette lotteries. That is, to each $s \in S$, an act assigns a roulette lottery $p \in L_{s}$. We formally define the set of acts $H$ as the Cartesian product, $\prod_{s \in S} L_{s}$. For each $h \in H$ and $p \in L_{s}$ denote by $\left(h^{-s}, p\right)$ the act obtained by replacing the $s$ coordinate of $h$ by $p$.

We view the set $H$ a mixture set since for any pair of acts $f, g \in H$ and any probability $\alpha \in[0,1]$ we shall identify $\alpha f+(1-\alpha) g$ with the act $h \in H$, for which $h_{s}=\alpha f_{s}+(1-\alpha) g_{s}$ for each $s \in S$.

In this two-stage formulation, after the first-stage, an agent knows which state $s$ in $S$ is the true state of nature, but unless the roulette lottery associated with that state is degenerate, she still does not know which prize in $X_{s}$ she will ultimately receive. We shall refer to this stage as the interim stage, where a typical interim-state is a state/roulette-lottery pair $(s, p)$ where $s \in S$ and $p \in L_{s}$. Correspondingly, the set of degenerate interim-states will be identified with the set of state-prize pairs $C=\left\{(s, x) \mid s \in S, x \in X_{s}\right\}$. In this case after the first stage, there is no more uncertainty left to be resolved.

Let $\otimes$ be a binary operation defined as follows: for all $\mu \in M$ and $f \in H, \mu \otimes f$ is the simple lottery defined on the set of interim states, that is, a simple interim-state lottery, in which for each $s \in S$ and $p \in L_{s}$,

$$
(\mu \otimes f)(s, p)=\left\{\begin{array}{cc}
\mu_{s} & \text { if } f_{s}=p \\
0 & \text { otherwise }
\end{array} .\right.
$$


An interim-state lottery may be viewed as an act (horse/roulette lottery) with preassigned probabilities, $\mu$, to the states (horses).

Let $\hat{L}$ denote the set of interim-state lotteries that can be expressed in this way. Notice that there exists a natural one-to-one correspondence between $\hat{L}$ and the set of simple probability measures on $C$. We can associate with each interim-state lottery, $\mu \otimes f$, the state-prize lottery $\ell$ defined by $\ell(s, x)=\mu_{s} f_{s}(x)$ for all $(s, x) \in C$. And with each state-prize lottery $\ell$ we can associate the interim-state lottery, $\mu \otimes f \in \hat{L}$, defined by $\mu_{s}=\sum_{x \in X_{s}} \ell(s, x)$ and

$$
f_{s}(x)=\left\{\begin{array}{cc}
0 & \text { if } \mu_{s}=0 \\
\ell(s, x) / \mu_{s} & \text { if } \mu_{s}>0
\end{array}, \text { for all } s \in S \text { and all } x \in X_{s} .\right.
$$

In addition to being able to express preferences between pairs of acts from $H$, we shall assume that an individual can also express preferences between pairs of interim-state lotteries from $\hat{L}$. Given the above one-to-one correspondence that exists between $\hat{L}$ and the set of simple probability measures on $C$, we shall sometimes identify this preference relation with the individual's preferences over state-prize lotteries.

Notice that $\delta^{s} \otimes f$ corresponds to the element of $\hat{L}$ that assigns to the interim-state $\left(s, f_{s}\right)$ the unit probability mass. We can thus express $\mu \otimes f=\sum_{s \in S} \mu_{s}\left(\delta^{s} \otimes f\right)$. More generally, we shall view the set $\hat{L}$ as a mixture space, since for any pair of probability measures $\mu, \nu \in M$, any pair of acts $f, g \in H$ and any probability $\alpha \in[0,1]$, we shall identify $\alpha(\mu \otimes f)+$ $(1-\alpha)(\nu \otimes g)$ with the interim-state lottery

$$
\sum_{s \in S}\left(\alpha \mu_{s}+(1-\alpha) \nu_{s}\right)\left(\delta^{s} \otimes\left(\frac{\alpha \mu_{s}}{\alpha \mu_{s}+(1-\alpha) \nu_{s}} f+\frac{(1-\alpha) \nu_{s}}{\alpha \mu_{s}+(1-\alpha) \nu_{s}} g\right)\right) .
$$

That is, each state $s \in S$, is assigned the probability weight $\alpha \mu_{s}+(1-\alpha) \nu_{s}$, and the roulette lottery in that state is given by

$$
\frac{\alpha \mu_{s}}{\alpha \mu_{s}+(1-\alpha) \nu_{s}} f_{s}+\frac{(1-\alpha) \nu_{s}}{\alpha \mu_{s}+(1-\alpha) \nu_{s}} g_{s}
$$

which is an element of $L_{s}{ }^{9}$

Preferences over the set of acts are given by $\succcurlyeq$, a binary relation on $H$. Preferences over the set of interim-state lotteries are given by $\hat{\succcurlyeq}$, a binary relation on $\hat{L}$. The asymmetric, $\succ$, $\hat{\succ}$ and symmetric, $\sim, \hat{\sim}$ parts of $\succcurlyeq$ and $\hat{\succcurlyeq}$, respectively, are defined as usual.

\footnotetext{
${ }^{9}$ Let $\ell$ (respectively, $\ell^{\prime}$ ) be the state-prize lottery associated with the interim-state lottery $\mu \otimes f$ (respectively, $\nu \otimes g)$. It is straightforward to check that for any $\alpha \in[0,1]$, the state-prize lottery associated with the interim-state lottery $\alpha(\mu \otimes f)+(1-\alpha)(\nu \otimes g)$ is $\alpha \ell+(1-\alpha) \ell^{\prime}$.
} 


\subsection{Axioms}

We require a non-degeneracy and richness for the state-prize space. Loosely speaking, we assume that in each state, there are at least two prizes between which the individual has a strict preference. That is, in each state $s$, there exist prizes $\bar{x}_{s}$ and $\underline{x}_{s}$ such that if the individual believed that state $s$ were going to obtain with probability one, he would strictly prefer receiving the prize $\bar{x}_{s}$ to receiving the prize $\underline{x}_{s}$. We can fix a set $\left\{\bar{x}_{s}, \underline{x}_{s}\right\}_{s \in S}$ and let $\bar{x}$ (respectively, $\underline{x}$ ) denote the act that yields in each state $s$, the degenerate roulette lottery $\delta^{\bar{x}_{s}}$ (respectively, $\delta \underline{\underline{x}}_{s}$ ). So that the preference relation over acts is not trivial, we assume that $\bar{x} \succ \underline{x}$. Furthermore, we require there exists the following 'preference overlap' between each pair of states. If the individual strictly prefers the state-prize $\left(t, \bar{x}_{t}\right)$ (that is, the better prize in state $t$ ) to the state-prize $\left(s, \bar{x}_{s}\right)$ (the better prize in state $s$ ), then he strictly prefers the state-prize $\left(s, \bar{x}_{s}\right)$ to $\left(t, \underline{x}_{t}\right)$ (the worse prize in state $t$ ) and he weakly prefers $\left(t, \underline{x}_{t}\right)$ to $\left(s, \underline{x}_{s}\right)$.

Formally we require:

(A.0) (State-Preference Overlap) There exists two acts $\bar{x}, \underline{x} \in H$, such that $\bar{x} \succ \underline{x}$ and for each $s \in S,\left(s, \bar{x}_{s}\right),\left(s, \underline{x}_{s}\right) \in C$ and $\delta^{s} \otimes \bar{x} \hat{\succ} \delta^{s} \otimes \underline{x}$. Furthermore, for every pair of states $s, t \in S$, if $\delta^{t} \otimes \bar{x} \hat{\zeta} \delta^{s} \otimes \bar{x}$ then $\delta^{s} \otimes \bar{x} \hat{\zeta} \delta^{t} \otimes \underline{x}$, and $\delta^{t} \otimes \underline{x} \hat{\succcurlyeq} \delta^{s} \otimes \underline{x}$.

Notice in particular, if two states each had only two possible prizes, state-preference overlap would imply that the 'better' and 'worse' prizes for one state could not be 'nested' in preference terms between the 'better' and 'worse' prize of the other state. That is, in the case where $X_{s}=\left\{\bar{x}_{s}, \underline{x}_{s}\right\}$ and $X_{t}=\left\{\bar{x}_{t}, \underline{x}_{t}\right\}$, the following preference ordering is not allowed: $\delta^{t} \otimes \bar{x} \hat{\zeta} \delta^{s} \otimes \bar{x} \hat{\zeta} \delta^{s} \otimes \underline{x} \hat{\succ} \delta^{t} \otimes \underline{x}$. To avoid possible misunderstanding consider the following two-state example in which each state has a continuum of prizes. $X_{1}=[1 / 4,3 / 4]$, $X_{2}=[0,1], \delta^{s} \otimes\left(f^{-s}, x\right) \hat{\succcurlyeq} \delta^{t} \otimes\left(f^{-t}, y\right)$, if and only if $x-y \geq 0$, and $f \succcurlyeq g$, if and only if

$$
\sum_{x \in[0,1]}\left(f_{1}(x)-g_{1}(x)+f_{2}(x)-g_{2}(x)\right) x \geq 0 .
$$

This example satisfies state-preference overlap even though the 'best' and 'worst' prizes for state 1 are nested between the 'best' and 'worst' prizes for state 2. For instance, if we take $\bar{x}_{1}:=3 / 4>\bar{x}_{2}:=1 / 2>\underline{x}_{1}:=1 / 4>\underline{x}_{2}:=0$, then we have the required preference overlap and $\bar{x} \succ \underline{x}$.

A similar requirement is imposed by Fishburn (1973) in his axiomatization of expected utility with state-dependent preferences. As Karni (1985) points out, this assumption may be irreconcilable with some applications of state-dependent preferences. A classic example would be in the context of flight insurance where the states of nature include both the 
states "life" and "death" and the prizes in each state are levels of wealth. In this case, state-preference overlap asserts that a passenger with no dependents may still prefer death over life if he takes out a sufficiently large insurance policy. Having said that, however, the state-preference overlap will be a maintained assumption throughout.

The next two assumptions are the standard ordering and continuity assumptions:

(A.1) $($ Weak Order) $\succcurlyeq$ and $\hat{\succcurlyeq}$ are complete and transitive.

(A.2) (Archimedean) (a) For all $f, g, h \in H$ such that $f \succ g \succ h$ there exist $\alpha, \beta \in(0,1)$ such that $\alpha f+(1-\alpha) h \succ g \succ \beta f+(1-\beta) h$. (b) For all $\mu \otimes f, \nu \otimes g, \eta \otimes h \in \hat{L}$ such that $\mu \otimes f \hat{\succ} \nu \otimes g \hat{\succ} \eta \otimes h$, there exist $\gamma, \varepsilon \in(0,1)$ such that $\gamma(\mu \otimes f)+(1-\gamma)(\eta \otimes h) \hat{\succ} \nu \otimes g$ $\hat{\succ} \varepsilon(\mu \otimes f)+(1-\varepsilon)(\eta \otimes h)$.

The first of our 'monotonicity' axioms, states that both preference relations $\succcurlyeq$ and $\hat{\succcurlyeq}$ respect a partial ordering of conditional first order stochastic dominance across prizes within a given state holding what happens off that state fixed. That is, moving probability from a conditionally less preferred prize to a conditionally more preferred prize within a state, but leaving the rest of the act or interim-state lottery unchanged makes the act or interim-state lottery more attractive.

(A.3) (Intra-State Monotonicity) For any state $s \in S$, any pair of prizes $x, y \in X_{s}$, any $\mu \in M$, any act $f \in H$, any roulette lottery $p \in L_{s}$, and any $\alpha \in(0,1]$ : (i) $\left(f^{-s}, x\right) \succcurlyeq(\succ)\left(f^{-s}, y\right)$ implies $\left(f^{-s}, \alpha \delta^{x}+(1-\alpha) p\right) \succcurlyeq(\succ)\left(f^{-s}, \alpha \delta^{y}+(1-\alpha) p\right)$, and

(ii) $\mu \otimes\left(f^{-s}, x\right) \succcurlyeq(\hat{\succ}) \mu \otimes\left(f^{-s}, y\right)$ implies $\mu \otimes\left(f^{-s}, \alpha \delta^{x}+(1-\alpha) p\right) \hat{\succcurlyeq}(\hat{\succ}) \mu \otimes\left(f^{-s}, \alpha \delta^{y}+(1-\alpha) p\right)$.

In the statement of the last axiom, nothing prevents the preference between two prizes in a given state, from depending upon the act (or, respectively, the interim-state lottery) in which they reside. The next axiom embodies a notion of ordinal act independence, that shall preclude such conditioning. That is, the ordinal ranking of prizes in a given state is independent of the act or interim-state lottery in which they reside. It does not imply, however, that the ordinal ranking of prizes is the same across states. For instance, if the prizes include tickets to a concert and an all-expenses paid skiing vacation, their ranking may be reversed depending on whether the states of nature are injured ankle or not. On the other hand if prizes are levels of wealth the ordinal ranking may be the same across states.

Ordinal act independence will have bite only if the state is question is obviously nonnull. 
Definition $1 A$ state $s$ is obviously null if $\mu \otimes\left(f^{-s}, p\right) \hat{\succ} \mu \otimes\left(f^{-s}, q\right)$ for some probability distribution $\mu \in M$, some roulette lotteries $p, q \in L_{s}$ and some act $f \in H$ but $\left(g^{-s}, p^{\prime}\right) \sim$ $\left(g^{-s}, q^{\prime}\right)$ for all roulette lotteries $p^{\prime}, q^{\prime} \in L_{s}$ and all acts $g \in H$. It is obviously nonnull if $\left(f^{-s}, p\right) \succ\left(f^{-s}, q\right)$ for some $f \in H$ and $p, q \in L_{s} .{ }^{10}$

(A.4) (Ordinal Act Independence) For any obviously nonnull state $s \in S$, any pair of prizes $x, y \in X_{s}$, any pair of acts $f, g \in H$ and any probability measure $\mu \in M$, such that $\mu_{s}>0: \mu \otimes\left(f^{-s}, x\right) \succcurlyeq \mu \otimes\left(f^{-s}, y\right)$ if and only if $\left(g^{-s}, x\right) \succcurlyeq\left(g^{-s}, y\right)$.

Ordinal act independence imposes the following consistency between the preference relations $\hat{\succcurlyeq}$ and $\succcurlyeq$. For each state $s$ in $S$, let $\hat{\succcurlyeq}_{s}$ and $\succcurlyeq_{s}$ be the preference relations on the prize space $X_{s}$ defined as follows: for all pairs of outcomes $x, y \in X_{s}$, if $\delta^{s} \otimes\left(\underline{x}^{-s}, x\right) \succcurlyeq \delta^{s} \otimes\left(\underline{x}^{-s}, y\right)$ then $x \hat{\succcurlyeq}_{s} y$, and if $\left(\underline{x}^{-s}, x\right) \succcurlyeq\left(\underline{x}^{-s}, y\right)$ then $x \succcurlyeq_{s} y$. Ordinal act independence and weak order imply that for every obviously nonnull state $s, \hat{\succcurlyeq}_{s}=\succcurlyeq_{s}$. Both binary relations inherit the weak ordering property of their respective 'parent' relations.

Given ordinal act independence it is meaningful to talk about monotonicity with respect to first order stochastic dominance for roulette lotteries within a given state.

Definition 2 For any state $s \in S$, and any pair of roulette lotteries $p=\left(x_{1}, p_{1} ; \ldots ; x_{m}, p_{m}\right) \in$ $L_{s}$ and $p^{\prime}=\left(x_{1}^{\prime}, p_{1}^{\prime} ; \ldots ; x_{m \prime}^{\prime}, p_{m \prime}^{\prime}\right) \in L_{s}$, the roulette lottery $p$ is said to first-order stochastically dominate on state $s$ the roulette lottery $p^{\prime}$ (written $p \geq_{s}^{1} p^{\prime}$ ), if

$$
\sum_{\left\{i: x_{i} \hat{\succcurlyeq}_{s} x_{k}^{\prime}\right\}} p_{i} \geq \sum_{\left\{j: x_{j}^{\prime} \hat{\succcurlyeq}_{s} x_{k}^{\prime}\right\}} p_{j}^{\prime} \text { for all } k=1, \ldots, m^{\prime} .
$$

The roulette lottery $p$ is said to strictly first-order stochastically dominate on state $s$ the roulette lottery $p^{\prime}$ (written $p>_{s}^{1} p^{\prime}$ ) if, in addition, strict inequality holds for some $k=$ $1, \ldots, m^{\prime}$.

The next lemma states that weak order, intra-state monotonicity and ordinal act independence together imply that if two acts (respectively, two interim-state lotteries) differ on only one obviously non-null state (respectively, on one state with positive probability), and for one act (respectively, interim-state lottery) the roulette lottery in that state (strictly) first-order

\footnotetext{
10 Notice that (A.0) and (A.3), imply that each state is either obviously null or obviously nonnull. The in between case, namely the case in which all the prizes in a given state are equally preferred is discussed in Karni, Schmeidler \& Vind (1983).
} 
stochastically dominates the roulette lottery on that state for the other act (respectively, interim-state lottery), then the former act (interim-state lottery) is (strictly) preferred to the latter.

Lemma 1 Suppose that $\succcurlyeq$ and $\hat{\succcurlyeq}$ satisfy (A.1), (A.3) and (A.4). Then, for any state $s \in S$, any pair of roulette lotteries $p, p^{\prime} \in L_{s}$, any act $f \in H$, and any $\mu \in M: p \geq_{s}^{1} p^{\prime}$ implies (i) $\left(f^{-s}, p\right) \succcurlyeq\left(f^{-s}, p^{\prime}\right)$ and (ii) $\mu \otimes\left(f^{-s}, p\right) \succcurlyeq \mu \otimes\left(f^{-s}, p\right)$ with strict preference in (i) (respectively, in (ii)) if $p>_{s}^{1} p^{\prime}$ and $s$ is obviously nonnull (respectively, $\mu_{s}>0$ ).

Given ordinal act independence, it is also meaningful to define monotonicity with respect to first order stochastic dominance for roulette lotteries across different states. That is, we can also introduce a first-order stochastic dominance partial ordering for degenerate interimstate lotteries where the dominance is with respect to the preference relation over $C$ induced by $\hat{\succcurlyeq}$.

Definition 3 For any pair of roulette lotteries $p \in L_{s}$ and $q \in L_{t}$, the degenerate interimstate lottery that yields $p$ on state $s$ is said to first-order stochastically dominate the degenerate interim-state lottery that yields $q$ on state $t$ (written $(s, p) \geq_{C}^{1}(t, q)$ ), if, for all $(r, x) \in C$

$$
\sum_{\left\{y \in X_{s}: \delta^{s} \otimes\left(h^{-s}, y\right) \succcurlyeq \delta^{r} \otimes\left(h^{-r}, x\right)\right\}} p(y) \geq \sum_{\left\{z \in X_{t}: \delta^{t} \otimes\left(h^{-t}, z\right) \succcurlyeq \delta^{r} \otimes\left(h^{-r}, x\right)\right\}} q(z) .
$$

Furthermore, the degenerate interim-state lottery that yields $p$ on state $s$ is said to strictly first-order stochastically dominate the degenerate interim-state lottery that yields $q$ on state $t$ (written $\left.(s, p)>_{C}^{1}(t, q)\right)$ if, in addition, strict inequality holds for some $(r, x) \in C$.

Our final monotonicity axiom requires that moving probability from states with firstorder stochastically inferior roulette lotteries to states with first-order stochastically superior roulette lotteries, increases the desirability of the interim-state lottery.

(A.5) (Inter-State Monotonicity) For any two states $s, t \in S$, any pair of roulette lotteries $p \in L_{s}, q \in L_{t}$, any act $f \in H$, and any pair of probability measures $\mu, \nu \in M$, if $(s, p) \geq_{C}^{1}(t, q), \nu_{s}>\mu_{s}$ and $\nu_{r}=\mu_{r}$ for all $r \in S-\{s, t\}$, then $\nu \otimes\left(\left(f^{-s}, p\right)^{-t}, q\right) \succcurlyeq \mu \otimes$ $\left(\left(f^{-s}, p\right)^{-t}, q\right)$. Furthermore, if $(s, p)>_{C}^{1}(t, q)$ then $\nu \otimes\left(\left(f^{-s}, p\right)^{-t}, q\right) \hat{\succ} \mu \otimes\left(\left(f^{-s}, p\right)^{-t}, q\right)$. 
As we have already observed above, ordinal act independence guarantees, for each obviously nonnull state $s \in S$, the agreement between the conditional preference relations $\hat{\succcurlyeq}_{s}$ and $\succcurlyeq_{s}$ induced on $X_{s}$ by $\hat{\succcurlyeq}$ and $\succcurlyeq$, respectively. But such consistency between $\hat{\succcurlyeq}$ and $\succcurlyeq$, although necessary for quantifiable beliefs, is not sufficient to ensure that it is possible to identify the preference relation over acts with the preference relation over interim-state lotteries restricted to a subset of such lotteries that have the same distribution over states. The next axiom, which is key to our theory, may be interpreted as setting the hurdle that must be cleared for us to be able to conclude that, at least, the preference between acts that differ in at most two states, is contained in the preference relation over interim-states lotteries, restricted to those that have a common distribution over states. To state this axiom we introduce the following additional notations and definitions: Fix some obviously nonnull state $s \in S$. For for every $t \in S-\{s\}$ and every $\mu \in M$, let $\alpha_{t}(\mu)$ be defined by

$\mu \otimes\left[\begin{array}{ccc}\bar{x}_{s} & \text { on } & s \\ \underline{x}_{t} & \text { on } & t \\ \underline{x}_{s^{\prime}} & \text { on } & s^{\prime} \notin S-\{s, t\}\end{array}\right] \hat{\sim} \mu \otimes\left[\begin{array}{ccc}{\left[\alpha_{t}(\mu) \delta^{\bar{x}_{s}}+\left(1-\alpha_{t}(\mu)\right) \delta \underline{x}_{s}\right]} & \text { on } & s \\ {\left[\alpha_{t}(\mu) \delta^{\bar{x}_{t}}+\left(1-\alpha_{t}(\mu)\right) \delta \underline{x}_{t}\right]} & \text { on } & t \\ \underline{x}_{s^{\prime}} & \text { on } & s^{\prime} \notin S-\{s, t\}\end{array}\right]$.

The correspondence $\alpha_{t}: M \rightarrow[0,1]$ defined above is, in fact, a function. This is an immediate implication of Lemma 4 below.

Let $\beta_{t}$ be defined by:

$$
\left[\begin{array}{ccc}
\bar{x}_{s} & \text { on } & s \\
\underline{x}_{t} & \text { on } & t \\
\underline{x}_{s^{\prime}} & \text { on } & s^{\prime} \notin S-\{s, t\}
\end{array}\right] \sim\left[\begin{array}{ccc}
{\left[\beta_{t} \delta^{\bar{x}_{s}}+\left(1-\beta_{t}\right) \delta \underline{x}_{s}\right]} & \text { on } & s \\
{\left[\beta_{t} \delta^{\bar{x}_{t}}+\left(1-\beta_{t}\right) \delta \underline{x}_{t}\right]} & \text { on } & t \\
\underline{x}_{s^{\prime}} & \text { on } & s^{\prime} \notin S-\{s, t\}
\end{array}\right] \text {. }
$$

To grasp the meaning of these definitions think of $\alpha_{t}(\mu)$ as an estimate of the probability of the state $s$ conditional on the event $\{s, t\}$ under $\mu$. Similarly, think of $\beta_{t}$ as an estimate of the decision-maker's subjective probability of state $s$ conditional on the event $\{s, t\}$. It is easy to verify that, if $\hat{\succcurlyeq}$ and $\succcurlyeq$ satisfy the axioms of expected utility and the corresponding von Neumann-Morgenstern utility functions are state-independent then these estimates are the true conditional probabilities (i.e., $\alpha_{t}(\mu)=\mu_{s} /\left(\mu_{s}+\mu_{t}\right)$ and if the subjective probability is denoted $\pi$ then $\left.\beta_{t}=\pi_{s} /\left(\pi_{s}+\pi_{t}\right)\right)$. In general, however, the estimates may deviate from the 
conditional probabilities. For instance, if $\hat{\succcurlyeq}$ and $\succcurlyeq$ satisfy the axioms of expected utility and the corresponding von Neumann-Morgenstern utility functions are state-dependent then,

$\alpha_{t}(\mu)=\frac{\mu_{s}\left[u_{s}\left(\bar{x}_{s}\right)-u_{s}\left(\underline{x}_{s}\right)\right]}{\mu_{s}\left[u_{s}\left(\bar{x}_{s}\right)-u_{s}\left(\underline{x}_{s}\right)\right]+\mu_{t}\left[u_{t}\left(\bar{x}_{t}\right)-u_{t}\left(\underline{x}_{t}\right)\right]}$ and $\beta_{t}=\frac{\pi_{s}\left[u_{s}\left(\bar{x}_{s}\right)-u_{s}\left(\underline{x}_{s}\right)\right]}{\pi_{s}\left[u_{s}\left(\bar{x}_{s}\right)-u_{s}\left(\underline{x}_{s}\right)\right]+\pi_{t}\left[u_{t}\left(\bar{x}_{t}\right)-u_{t}\left(\underline{x}_{t}\right)\right]}$.

If the utility functions representing the preferences relations $\hat{\succcurlyeq}$ and $\succcurlyeq$ are not linear in the probabilities then the estimates may depend on the entire vector of payoffs.

The next axiom requires that whenever these estimates are in agreement (i.e., when $\mu$ satisfies $\left.\alpha_{t}(\mu)=\beta_{t}\right)$ then the underlying preference relations $\hat{\succcurlyeq}$ and $\succcurlyeq$ must be in agreement on the ranking of acts that differ only on the conditioning (two-state) event, $\{s, t\}$, and the ranking of the corresponding interim-state lotteries.

The acts $f$ and $g$ are said to agree outside the event $\{s, t\}$ if $f_{s^{\prime}}=g_{s^{\prime}}$ for all $s^{\prime} \notin S-\{s, t\}$. With these definitions in mind we state the concordance axiom:

(A.6) (Concordance) Fix some obviously nonnull state $s \in S$ then, for every $t \in S-\{s\}$, every probability measure $\mu \in M$, and every $f, g \in H$ that agree outside the event $\{s, t\}$, if $\beta_{t}=\alpha_{t}(\mu)$ then

$$
\mu \otimes f \hat{\succcurlyeq} \mu \otimes g \Leftrightarrow f \succcurlyeq g
$$

The next lemma contains a critical observation, namely, that for some probability measure $\mu \in M, \beta_{t}=\alpha_{t}(\mu)$ for all $t \in S-\{s\}$. Thus, the concordance axiom is not vacuous.

Lemma 2 There exist $\mu \in M$ such that $\beta_{t}=\alpha_{t}(\mu)$ for all $t \in S-\{s\}$.

\subsection{Preliminary Results}

The following results will be used in the proof of the main representation theorem. To facilitate the exposition we introduce the following notations and definitions. First, we say $f$ (strictly) first-order stochastically dominates $g$ state-wise, written $f \geq_{H}^{1}\left(>_{H}^{1}\right) g$, if $f_{s} \geq_{s}^{1} g_{s}$ for all $s \in S$ (and with $f_{t}>_{t}^{1} g_{t}$ for some obviously nonnull $t \in S$ ). Next we define a partial order, $\geq_{\hat{L}}$, on the set of interim-state lotteries as follows: if $f \geq_{H}^{1} g$ then for any $\mu \in M$, we write $\mu \otimes f \geq \hat{\hat{L}}^{1} \mu \otimes g$; and, for any act $f \in H$ and any pair of states, $s, t \in S$, if $\left(s, f_{s}\right) \geq_{C}^{1}\left(>_{C}^{1}\right)\left(t, f_{t}\right)$ then, for any $\mu, \nu \in M$, such that $\mu_{s}>\nu_{s}$, and $\mu_{s^{\prime}}=\nu_{s^{\prime}}$ for all $s^{\prime} \in S-\{s, t\}$, we write $\mu \otimes f \geq_{\hat{L}}^{1} \nu \otimes f$. Let $\geq_{\hat{L}}$ be the transitive closure of $\geq_{\hat{L}}^{1}$, and let $>_{\hat{L}}$ denote the asymmetric part of $\geq_{\hat{L}}$. If $\mu \otimes f \geq_{\hat{L}} \nu \otimes g$, then we shall say that the 
interim-state lottery $\mu \otimes f$ indirectly first-order stochastically dominates the interim-state lottery $\nu \otimes g$. Similarly, if $\mu \otimes f>_{\hat{L}} \nu \otimes g$ then we shall say that $\mu \otimes f$ indirectly strictly first-order stochastically dominates $\nu \otimes g$.

Definition 4 (a) The binary relation $\succcurlyeq$ satisfies mixture monotonicity if, for all $f, g \in H$ such that $f>_{H}^{1} g$, and all $\alpha, \beta \in[0,1]$ :

$$
\alpha \geq \beta \Leftrightarrow \alpha f+(1-\alpha) g \succcurlyeq \beta f+(1-\beta) g \text {. }
$$

(b) The binary relation $\hat{\succcurlyeq}$ satisfies mixture monotonicity if, for all $f, g \in H, \mu, \nu \in M$ such that $\mu \otimes f>_{\hat{L}} \nu \otimes g$, and $\alpha, \beta \in[0,1]:$

$$
\alpha \geq \beta \Leftrightarrow \alpha(\mu \otimes f)+(1-\alpha)(\nu \otimes g) \succcurlyeq \beta(\mu \otimes f)+(1-\beta)(\nu \otimes g) .
$$

Lemma 3 (Mixture Monotonicity) Axioms A.1, and (A.3)-(A.5) imply that $\succcurlyeq$ and $\hat{\succcurlyeq}$ satisfy mixture monotonicity.

Definition 5 (a) The binary relation $\succcurlyeq$ satisfies mixture solvability if for all $f, g, h \in H$ such that $f>_{H}^{1} g$, and $f \succcurlyeq h \succcurlyeq g$, there exists a unique $\alpha \in[0,1]$ such that $\alpha f+(1-\alpha) g \sim h$.

(b) The binary relation $\succcurlyeq$ satisfies mixture solvability if for all $f, g, h \in H$, and for all $\mu, \nu, \eta \in M$ such that $\mu \otimes f>_{\hat{L}} \nu \otimes g$, and $\mu \otimes f \succcurlyeq \eta \otimes h \succcurlyeq \nu \otimes g$, there exists a unique $\beta \in[0,1]$ such that $\beta(\mu \otimes f)+(1-\beta)(\nu \otimes g) \hat{\sim} \eta \otimes h$.

Lemma 4 (Mixture Solvability) Axioms (A.1)-(A.5) imply that $\succcurlyeq$ and $\hat{\succcurlyeq}$ satisfy mixture solvability.

For a proof see Fishburn (1970, Lemma 3.1 p.33) 


\section{Representation}

\subsection{Concordance Extended}

As a preliminary step toward the statement of our main representation theorem we show, in the next lemma, that the agreement between the preference relation over acts and the restricted preference relation over interim-state lotteries imposed by the concordance axiom is sufficient to ensure that these two relations agree over the entire set of acts.

Lemma 5 Assume that $\succcurlyeq$ and $\hat{\succcurlyeq}$ satisfy (A.1) - (A.6) and let $\mu^{*} \in M$ be such that $\mu_{s}^{*}>0$ and $\beta=\alpha\left(\mu^{*}\right)$. Then for each triple of acts $f, g, h \in H$, for which $g>_{H}^{1} h$ and $g \geq_{H}^{1} f \geq_{H}^{1} h$, and for every event $E \subset S$, for which $s$ is an element, there exists a unique $\alpha \in[0,1]$, such that

$$
\mu^{*} \otimes f \hat{\sim} \mu^{*} \otimes\left[\begin{array}{cc}
\alpha g_{t}+(1-\alpha) h_{t} & t \in E \\
f_{s^{\prime}} & s^{\prime} \notin E
\end{array}\right] \Leftrightarrow f \sim\left[\begin{array}{cc}
\alpha g_{t}+(1-\alpha) h_{t} & t \in E \\
f_{s^{\prime}} & s^{\prime} \notin E
\end{array}\right] .
$$

\subsection{The Main Theorem}

Each function $V: \hat{L} \rightarrow \mathbb{R}$ induces a preference relation on $\hat{L}$. In particular, it induces a preference relation over $C$, the set of state-prize pairs. Hence we can define the partial ordering over degenerate interim-state lotteries of first-order stochastic dominance with respect to the preference relation over $C$ that is induced by $V$.

Definition 6 Fix a function $V: \hat{L} \rightarrow \mathbb{R}$. For any pair of roulette lotteries $p \in L_{s}$ and $q \in L_{t}$, the degenerate interim-state lottery that yields $p$ on state $s$ is said to first-order stochastically dominate with respect to the order on $C$ induced by $V$ the degenerate interim-state lottery that yields $q$ on state $t$ (written $(s, p) \geq_{V}^{1}(t, q)$ ) if, for all $(r, x) \in C$,

$$
\sum_{\left\{y \in X_{s}: V\left(\delta^{s} \otimes\left(\underline{x}^{-s}, y\right)\right) \geq V\left(\delta^{r} \otimes\left(\underline{x}^{-r}, x\right)\right)\right\}} p(y) \geq \sum_{\left\{z \in X_{t}: V\left(\delta^{t} \otimes\left(\underline{x}^{-t}, z\right)\right) \geq V\left(\delta^{r} \otimes\left(\underline{x}^{-r}, x\right)\right)\right\}} q(z) .
$$

Furthermore, $p$ (on state $s$ ) is said to strictly first-order stochastically dominate with respect to the order on $C$ induced by $V$ the degenerate interim-state lottery that yields $q$ on state $t$ (written $\left.(s, p)>_{V}^{1}(t, q)\right)$, if, in addition, strict inequality holds for some $(r, x) \in C$.

Using definition 6 we define a partial order on $\hat{L}$, induced by $V$, that we refer to as $V$-dominance. 
Definition 7 Fix a function $V: \hat{L} \rightarrow \mathbb{R}$. Set $\geq_{V}$ equal to the union of the following two sets:

1. $\left\{(\mu \otimes f, \mu \otimes g): \mu \in M, f, g \in H,\left(s, f_{s}\right) \geq_{V}^{1}\left(s, g_{s}\right)\right.$, for all $\left.s \in S\right\}$

2. $\left\{(\nu \otimes f, \mu \otimes f): \mu, \nu \in M, f \in H\right.$, s.t. $\nu_{s}>\mu_{s}$ and $\mu_{s^{\prime}}=\nu_{s^{\prime}}$, for all $\left.s^{\prime} \notin\{s, t\},\left(s, f_{s}\right) \geq_{V}^{1}\left(t, f_{t}\right)\right\}$

Set $\geq_{\hat{L}}^{V}$ equal to the transitive closure of $\geq_{V}$ and set $>_{\hat{L}}^{V}$ equal to the asymmetric part of $\geq{ }_{\hat{L}}^{V}$

Our notion of monotonicity for $V$ is the functional analog of the conjunction of intrastate monotonicity, ordinal act independence and inter-state monotonicity for a preference relation defined over $\hat{L}$. It says that if $\mu \otimes f$ (strictly) $V$-dominates $\nu \otimes g$ then $V(\mu \otimes f) \geq$ (>) $V(\nu \otimes g)$. Formally:

Definition 8 A function $V: \hat{L} \rightarrow \mathbb{R}$ is said to be strictly monotonic if, for all pairs of interim-state lotteries, $\mu \otimes f, \nu \otimes g \in \hat{L}, \mu \otimes f \geq_{\hat{L}}^{V}\left(>_{\hat{L}}^{V}\right) \nu \otimes g$ implies $V(\mu \otimes f) \geq(>)$ $V(\nu \otimes g)$.

With this notion of monotonicity in mind we are in a position to state our main result. A function $F: D \rightarrow \mathbb{R}$ is said to represent the binary relation $\succcurlyeq$ on $D$ if, for all $y, z \in D$, $y \succcurlyeq z \Leftrightarrow F(y) \geq F(z)$. Moreover, if $D$ is a mixture space, $F$ is said to be mixture continuous if $F(\alpha y+(1-\alpha) z)$ is continuous in $\alpha$ for all $y, z \in D$.

Theorem 1 Let $\succcurlyeq$ and $\hat{\succcurlyeq}$ be binary relations on $H$ and $\hat{L}$, respectively, satisfying statepreference overlap. Then the following conditions are equivalent:

(i) The binary relations $\succcurlyeq$ and $\hat{\succcurlyeq}$ satisfy, weak-order, Archimedean, intra-state monotonicity for acts, ordinal act independence, inter-state monotonicity and concordance.

(ii) There exists a unique probability measure $\pi$ on $S$ such that $\pi_{s}=0$ if and only if $s$ is obviously null and a mixture continuous, strictly monotonic function $V: \hat{L} \rightarrow \mathbb{R}$ such that:

(a) V represents $\hat{\succcurlyeq}$

(b) For all $f, g \in H, f \succcurlyeq g \Leftrightarrow V(\pi \otimes f) \geq V(\pi \otimes g)$. 


\section{Special Cases}

\subsection{Expected utility theory}

A special case of our theory of quantifiable beliefs is obtained if the preference relations $\succcurlyeq$ and $\hat{\succcurlyeq}$ satisfy the independence axiom of expected utility theory. Formally,

(A.7) (Independence) For all $f, g, h \in H, \nu, \mu, \eta \in M$, and $\alpha \in(0,1]$,

(i) $f \succcurlyeq g \Leftrightarrow \alpha f+(1-\alpha) h \succcurlyeq \alpha g+(1-\alpha) h$.

(ii) $\nu \otimes f \succcurlyeq \mu \otimes g \Leftrightarrow \alpha(\nu \otimes f)+(1-\alpha)(\eta \otimes h) \succcurlyeq \alpha(\mu \otimes g)+(1-\alpha)(\eta \otimes h)$.

Suppose that the preference relation $\hat{\succcurlyeq}$ satisfies weak-order, Archimedean, and independence then, by the von Neumann-Morgenstern theorem, the utility representation of $\hat{\succcurlyeq}$ is affine and thus there exists a utility function $u: C \rightarrow \mathbb{R}$ such that

$$
\nu \otimes f \hat{\sim} \mu \otimes g \Leftrightarrow \sum_{s \in S} \sum_{x \in X_{s}} u(s, x) \nu_{s} f_{s}(x)=\sum_{s \in S} \sum_{x \in X_{s}} u(s, x) \mu_{s} g_{s}(x) .
$$

Moreover, it is easy to verify that the independence axiom implies intra-state monotonicity for acts (A.3), inter-state monotonicity (A.5) and, together with concordance, it also implies ordinal act independence (A.4).

Let $f$ and $g$ be two acts that agree outside a state $s$ (i.e., $f_{t}=g_{t}$ for all $t \in S-\{s\}$.) Then, by Lemma 5 and independence (which implies mixture monotonicity) if, for some $\mu \in M$ such that $\mu_{s}>0$, and obviously nonnull $s \in S$,

$$
\mu \otimes f \succcurlyeq \mu \otimes g \Leftrightarrow f \succcurlyeq g
$$

then, by the expected utility representation, for all $\nu \in M$ such that $\nu_{s}>0$,

$$
\sum_{x \in X_{s}} u(s, x) \nu_{s} f_{s}(x) \geq \sum_{x \in X_{s}} u(s, x) \nu_{s} g_{s}(x) \Rightarrow \sum_{x \in X_{s}} u(s, x) f_{s}(x) \geq \sum_{x \in X_{s}} u(s, x) g_{s}(x) .
$$

For obviously nonnull states the reverse implication also holds. Since $\nu \otimes f$ and $\nu \otimes g$ agree outside $s$ this implies that,

$$
\nu \otimes f \succcurlyeq \nu \otimes g \Rightarrow f \succcurlyeq g
$$

and for obviously nonnull $s \in S$

$$
\nu \otimes f \succcurlyeq \nu \otimes g \Leftrightarrow f \succcurlyeq g .
$$


But the last two conditions are equivalent to the axiom of strong concordance introduced by Karni and Schmeidler (1981). Consequently, assuming independence, our main result implies the theory of Karni and Schmeidler (1981) as a special case. Formally,

Theorem 2 (Karni and Schmeidler (1981).) Let $\succcurlyeq$ and $\hat{\succcurlyeq}$ be binary relations on $H$ and $\hat{L}$, respectively, that satisfy state-preference overlap. Then the following conditions are equivalent:

(i) Each of the preference relations $\succcurlyeq$ and $\succcurlyeq$ satisfy (A.1), (A.2), (A.7) and jointly they satisfy (A.6).

(ii) There exists a real-valued function $u$ on $\left\{(s, x) \mid s \in S, x \in X_{s}\right\}$ and a probability measure $\pi \in M$ such that, for all $f, g \in H$

$$
f \succcurlyeq g \Leftrightarrow \sum_{s \in S} \pi_{s} \sum_{x \in X_{s}} u(s, x) f_{s}(x) \geq \sum_{s \in S} \pi_{s} \sum_{x \in X_{s}} u(s, x) g_{s}(x)
$$

and, for all $\mu \otimes f, \nu \otimes g \in \hat{L}$

$$
\mu \otimes f \hat{\succcurlyeq} \nu \otimes g \Leftrightarrow \sum_{s \in S} \sum_{x \in X_{s}} u(s, x) \mu_{s} f_{s}(x) \geq \sum_{s \in S} \sum_{x \in X_{s}} u(s, x) \mu_{s} g_{s}(x)
$$

Moreover, (a) $\pi$ is unique and $\pi(s)=0$ if and only if is obviously null, and (b) $u$ is unique up to cardinal unit-comparable transformation.

If we assume, in addition, that $\succcurlyeq$ and $\succcurlyeq$ satisfy state-independence then we get a version of the theory of Anscombe and Aumann (1963). Specifically, let $X_{s}=X$ for all $s \in S$, and assume:

(A.8) (State-independence) For all $h \in H, \nu \in M$ and $p, q \in L$,

(i) If for some obviously nonnull state $s \in S,\left(h^{-s}, p\right) \succcurlyeq\left(h^{-s}, q\right)$ then $\left(h^{-t}, p\right) \succcurlyeq$ $\left(h^{-t}, q\right)$ for every $t \in S$.

(ii) If for some $s \in S$ such that $\nu_{s}>0, \nu \otimes\left(h^{-s}, p\right) \succcurlyeq \nu \otimes\left(h^{-s}, q\right)$ then $\nu \otimes\left(h^{-t}, p\right)$ $\hat{\succcurlyeq} \nu \otimes\left(h^{-t}, q\right)$ for every state $t \in S$.

Clearly, the KS theorem implies a subjective expected utility representations. Specifically, the following follows directly from the preceding theorem. 
Corollary 1 Let $\succcurlyeq$ and $\hat{\succcurlyeq}$ be binary relations on $H$ and $\hat{L}$. Suppose that $X_{s}=X$ for each $s \in S$ and $\succ$ is non-empty. ${ }^{11}$ Then the following conditions are equivalent:

(i) Each of the preference relations $\succcurlyeq$ and $\succcurlyeq$ satisfy (A.1), (A.2), (A.7), (A.8) and jointly they satisfy (A.6).

(ii) There exists a real-valued function $u$ on $X$, a probability measure $\pi \in M$, and $b \in \mathbb{R}_{+}^{S}$ such that, for all $f, g \in H$

$$
f \succcurlyeq g \Leftrightarrow \sum_{s \in S} \pi_{s} \sum_{x \in X_{s}} b_{s} u(x) f_{s}(x) \geq \sum_{s \in S} \pi_{s} \sum_{x \in X_{s}} b_{s} u(x) g_{s}(x)
$$

and, for all $\mu \otimes f, \nu \otimes g \in \hat{L}$

$$
\mu \otimes f \hat{\succcurlyeq} \otimes \theta \Leftrightarrow \sum_{s \in S} \sum_{x \in X_{s}} b_{s} u(x) \mu_{s} f_{s}(x) \geq \sum_{s \in S} \sum_{x \in X_{s}} b_{s} u(x) \nu_{s} g_{s}(x) .
$$

Moreover, (a) $\pi$ is unique and $\pi(s)=0$ if and only if is obviously null, and (b) $u$ is unique up to positive linear transformation and $b$ is unique.

Notice that this representation differs from that of Anscombe and Aumann (1963) in that the evaluation of outcomes, but not the ranking of roulette lotteries, may vary across states. Thus, it may be the case that $b_{s} \neq b_{t}$. In this case, AA will redefine the state probabilities as follows:

$$
\bar{\pi}_{s}=\frac{\pi_{s} b_{s}}{\sum_{t \in S} \pi_{t} b_{t}}, \text { for all } s \in S
$$

to obtain the representation

$$
f \succcurlyeq g \Leftrightarrow \sum_{s \in S} \bar{\pi}_{s} \sum_{x \in X_{s}} u(x) f_{s}(x) \geq \sum_{s \in S} \bar{\pi}_{s} \sum_{x \in X_{s}} u(x) g_{s}(x) .
$$

Thus, except for the case in which $b_{s}=b$ for all $s$, the AA subjective probabilities misrepresents the decision-makers' beliefs whereas quantifiable beliefs theory, since it implies the KS model, identifies the correct probability representation of beliefs. ${ }^{12}$

\footnotetext{
11 Notice that the hypothesis of the theorem implies (A.0).

12 A more elaborate discussion of this point as well as the methodological issues it raises is provided in Karni and Mongin (2000).
} 


\subsection{Probabilistic Sophistication}

As already noted, the MS definition of probabilistic sophistication entails two implicit assumptions, namely, reduction of compound lotteries and state-independence. We show next that, under these additional assumptions, the theory of quantifiable beliefs implies a version of MS probabilistic sophistication. Furthermore, as is the case of the KS versus the AA definition of subjective probabilities, if the theory of quantifiable beliefs and the theory of probabilistic sophistication yield diverse subjective probabilities then the notion of subjective probabilities corresponding to quantifiable beliefs is a correct representation of the decisionmakers' beliefs while that of probabilistic sophistication is not. In other words, suppose we start with a given probability distribution over the set of states that we know represent the decision-maker's beliefs and on the basis of these probabilities induce a preference relation on acts that satisfies the requirements of probabilistic sophistication. Whereas the probabilities that figure in the theory of quantifiable beliefs are those that represent the decision-maker's beliefs, this is not necessarily true of the probabilities that figure in the MS model. A specific example in given below.

Machina and Schmeidler (1995) embed their theory in the analytical framework of AA. ${ }^{13}$ They postulate a preference relation on the set of acts, $H$, and assume that, for all $s \in S$, $X_{s}=X, L_{s}=L$, and that $X$ contains a best and worst outcomes, denoted by $M$ and 0 , respectively. ${ }^{14}$ We assume, therefore, that these restrictions hold in our model.

We observe that Lemma 1 implies that intra-state monotonicity and ordinal act independence imply MS axiom 5 which they refer to as First-Order Stochastic Dominance Preference.

To conform with the MS definition of probabilistic sophistication, we assume a 'stateindependent reduction of compound lotteries' property. To facilitate the exposition we introduce the following additional notations: (i) for each $x \in X$, let $x$ also denote the constant act $f$, for which $f_{s}=\delta^{x}$ for all $s \in S$; (ii) for each $\mu \otimes f \in \hat{L}$ denote by $(\mu \otimes f)_{m}$ its marginal distribution on $X$ (i.e., for each $x \in X,(\mu \otimes f)_{m}(x)=\sum_{s \in S} \mu_{s} f_{s}(x)$.) We shall also introduce a first-order stochastic dominance partial ordering for distributions on $X$ where the dominance is with respect to the restriction of $\succcurlyeq$ to constant acts that yield a degenerate roulette lottery.

\footnotetext{
13 The MS model does not require the state-space to be finite. Our treatment, however, is confined to the case of a finite state space.

14 To be exact, Machina and Schmeidler define a preference relation on a set that includes pure roulette, pure horse lotteries, and horse/roulette lotteries. However, pure roulette lotteries are constant acts with nondegenerate roulette lotteries as outcomes, pure horse lotteries are acts with degenerate lotteries as outcomes, both of these are specific cases of horse/roulette lotteries, which are acts in our terminology.
} 
Definition 9 For any pair of interim-state lotteries $\mu \otimes f$ and $\nu \otimes g \in \hat{L},(\mu \otimes f)_{m}$ is said to first-order stochastically dominate $(\nu \otimes g)_{m}$ (written $\left.(\mu \otimes f)_{m} \geq_{X}^{1}(\nu \otimes g)_{m}\right)$, if

$$
\sum_{\{y: y \succcurlyeq x\}}(\mu \otimes f)_{m}(y) \geq \sum_{\{y: y \succcurlyeq x\}}(\nu \otimes g)_{m}(y) \text { for all } x \in X
$$

Furthermore, $(\mu \otimes f)_{m}$ is said to strictly first-order stochastically dominate $(\nu \otimes g)_{m}$ (written $\left.(\mu \otimes f)_{m}>_{X}^{1}(\nu \otimes g)_{m}\right)$ if, in addition, strict inequality holds for some $x \in X$.

In our framework, the MS notion of probabilistic sophistication requires, in addition, the following:

(A.9) For all $\mu \in M$ and all $f, g \in H,(\mu \otimes f)_{m} \geq_{X}^{1}\left(>_{X}^{1}\right)(\mu \otimes g)_{m}$ implies $\mu \otimes f \succcurlyeq(\hat{\succ})$ $\mu \otimes g$

The next lemma shows that (A.9) implies both intra-state monotonicity and inter-state monotonicity hold for $\hat{\succcurlyeq}$.

Lemma 6 Suppose that $\succcurlyeq$ and $\hat{\succcurlyeq}$ jointly satisfy (A.4) and (A.9). Then, $\hat{\succcurlyeq}$ satisfies (A.3) and (A.5).

Clearly, (A.9) is not implied by the theory of quantifiable beliefs. However, if we impose (A.9) as an additional condition then the theory of quantifiable beliefs implies MS axiom 6 and hence probabilistic sophistication.

Fix a partition $\left\{E_{1}, \ldots, E_{n}\right\}$ of $S$ and suppose that for some $\alpha \in[0,1]$ and a pair of events, $E_{i}, E_{j}$ the following condition holds: ${ }^{15}$

$$
h \equiv\left[\begin{array}{ccc}
\delta^{M} & \text { on } & E_{i} \\
\delta^{0} & \text { on } & E_{j} \\
\delta^{0} & \text { on } & E_{k}, k \neq i, j
\end{array}\right] \sim\left[\begin{array}{ccc}
\alpha \delta^{M}+(1-\alpha) \delta^{0} & \text { on } & E_{i} \\
\alpha \delta^{M}+(1-\alpha) \delta^{0} & \text { on } & E_{j} \\
\delta^{0} & \text { on } & E_{k}, k \neq i, j
\end{array}\right] \equiv h^{\prime} .
$$

\footnotetext{
${ }^{15}$ This is, in our notations, the condition in the hypothesis of Machina and Schmeidler (1995) axiom 6.
} 
By Theorem 1 (1) is equivalent to the following condition:

$\pi \otimes h \equiv \pi \otimes\left[\begin{array}{cccc}\delta^{M} & \text { on } & E_{i} \\ \delta^{0} & \text { on } & E_{j} \\ \delta^{0} & \text { on } & E_{k}, k \neq i, j\end{array}\right] \hat{\sim} \pi \otimes\left[\begin{array}{ccc}\alpha \delta^{M}+(1-\alpha) \delta^{0} & \text { on } & E_{i} \\ \alpha \delta^{M}+(1-\alpha) \delta^{0} & \text { on } & E_{j} \\ \delta^{0} & \text { on } & E_{k}, k \neq i, j\end{array}\right] \equiv \pi \otimes h^{\prime}$.

But,

$$
(\pi \otimes h)_{m}=\left[M, \pi\left(E_{i}\right) ; 0,\left(1-\pi\left(E_{i}\right)\right)\right]
$$

and

$$
\left(\pi \otimes h^{\prime}\right)_{m}=\left[M, \alpha\left(\pi\left(E_{i}\right)+\pi\left(E_{j}\right)\right) ; 0,\left(1-\alpha\left(\pi\left(E_{i}\right)+\pi\left(E_{j}\right)\right)\right] .\right.
$$

Hence, $\pi \otimes h \hat{\sim} \pi \otimes h^{\prime}$ and (A.9) imply that

$$
\alpha=\frac{\pi\left(E_{i}\right)}{\pi\left(E_{i}\right)+\pi\left(E_{j}\right)} .
$$

That is, $(\pi \otimes h)_{m}=\left(\pi \otimes h^{\prime}\right)_{m}$.

Next consider the interim-state lotteries

$\pi \otimes f \equiv \pi \otimes\left[\begin{array}{ccc}p & \text { on } & E_{i} \\ q & \text { on } & E_{j} \\ r_{k} & \text { on } & E_{k}, k \neq i, j\end{array}\right]$ and $\pi \otimes g \equiv \pi \otimes\left[\begin{array}{ccc}\alpha p+(1-\alpha) q & \text { on } & E_{i} \\ \alpha p+(1-\alpha) q & \text { on } & E_{j} \\ r_{k} & \text { on } & E_{k}, k \neq i, j\end{array}\right]$.

Observe that for all $x \in X$

$$
(\pi \otimes f)_{m}(x)=\pi\left(E_{i}\right) p(x)+\pi\left(E_{j}\right) q(x)+\sum_{k \neq i, j} \pi\left(E_{k}\right) r_{k}(x)
$$

and

$$
(\pi \otimes g)_{m}(x)=\left(\pi\left(E_{i}\right)+\pi\left(E_{j}\right)\right)(\alpha p(x)+(1-\alpha) q(x))+\sum_{k \neq i, j} \pi\left(E_{k}\right) r_{k}(x) .
$$


Since $\alpha=\pi\left(E_{i}\right) /\left(\pi\left(E_{i}\right)+\pi\left(E_{j}\right)\right)$, the last two equations imply $(\pi \otimes f)_{m}=(\pi \otimes g)_{m}$. Hence, by (A.9),

$$
\pi \otimes\left[\begin{array}{ccc}
p & \text { on } & E_{i} \\
q & \text { on } & E_{j} \\
r_{k} & \text { on } & E_{k}, k \neq i, j
\end{array}\right] \hat{\sim} \pi \otimes\left[\begin{array}{ccc}
\alpha p+(1-\alpha) q & \text { on } & E_{i} \\
\alpha p+(1-\alpha) q & \text { on } & E_{j} \\
r_{k} & \text { on } & E_{k}, k \neq i, j
\end{array}\right] .
$$

But, by Theorem 1 and concordance, the last statement is equivalent to

$$
\left[\begin{array}{ccc}
p & \text { on } & E_{i} \\
q & \text { on } & E_{j} \\
r_{k} & \text { on } & E_{k}, k \neq i, j
\end{array}\right] \sim\left[\begin{array}{ccc}
\alpha p+(1-\alpha) q & \text { on } & E_{i} \\
\alpha p+(1-\alpha) q & \text { on } & E_{j} \\
r_{k} & \text { on } & E_{k}, k \neq i, j
\end{array}\right] .
$$

We showed that (1) implies (2) which is the Horse/Roulette Replacement Axiom, axiom 6, of MS.

We may now state the following result:

Theorem 3 Let $\succcurlyeq$ and $\hat{\succcurlyeq}$ be binary relations on $H$ and $\hat{L}$. Suppose that $X_{s}=X$ for each $s \in S, \succ$ is non-empty and there exists a best and worst outcome. Then the following conditions are equivalent:

(i) Both preference relations $\succcurlyeq$ and $\hat{\succcurlyeq}$ satisfy (A.1) and (A.2). Furthermore, $\succcurlyeq$ satisfies (A.3), $\succcurlyeq$ satisfies (A.9) and jointly they satisfy (A.4) and (A.6).

(ii) There exists a real-valued function $V: L \rightarrow \mathbb{R}$ and a probability measure $\pi \in M$,

$$
f \succcurlyeq g \Leftrightarrow V\left(\sum_{s \in S} \pi_{s} f_{s}\right) \geq V\left(\sum_{s \in S} \pi_{s} g_{s}\right)
$$

and, for all $\mu \otimes f, \nu \otimes g \in \hat{L}$

$$
\mu \otimes f \hat{\succcurlyeq} \otimes g \Leftrightarrow V\left(\sum_{s \in S} \mu_{s} f_{s}\right) \geq V\left(\sum_{s \in S} \nu_{s} g_{s}\right) .
$$

Moreover, $\pi$ is unique and $\pi(s)=0$ if and only if is obviously null.

The theory of probabilistically sophisticated choice is a special case of quantifiable beliefs theory. However, there are instances in which, analogously to the conflict between the KS and 
the AA definitions of subjective probabilities, the two theories agree on the preferences over acts and yet yield different probabilities measures on set of states. This is due to the fact that the state-independence axiom (A.9) is an inherent property of probabilistic sophistication but is not necessarily a property of quantifiable beliefs theory. Suppose that there are $\mu \otimes f$ and $\nu \otimes g$ in $\hat{L}$ such that $(\mu \otimes f)_{m}=(\nu \otimes g)_{m}$ and yet $\mu \otimes f \hat{\succ} \nu \otimes g$. Since the theory of probabilistically sophisticated choice does not "see" $\hat{\succ}$ and is not concerned with this auxiliary preference relation, it forces the subjective probabilities to be consistent with (A.9) even though these probabilities imply $\mu \otimes f \hat{\sim} \nu \otimes g$. Hence, the notion of subjective probabilities corresponding to probabilistically sophisticated choice may misrepresent the decision-maker's beliefs. Recall that the AA theory is a special case of probabilistic sophistication and the KS theory is a specific case of quantifiable beliefs. For expected utility theory this point is made at the end of the preceding subsection. The following example shows that the problem is not confined to subjective expected utility theory.

Example 1 Let $S=\{1,2\}, X_{1}=X_{2}=[0,2]$ and consider a decision-maker who believes that the two states are equally likely to occur. Suppose further that his preferences over $\hat{L}$ is represented by a weighted utility functional with state-independent utility function $u(s, x)=u(x)=x$ and state-dependent weight function $\hat{w}(s, x)=b(s) w(x)$, where $b(s)=$ $s, w(x)=1 /(1+x)$. Let $f$ and $g$ be "pure horse lotteries" given by: $f(1)=1, f(2)=1$, $g(1)=0$ and $g(2)=2$. Then, given the decision-maker's beliefs $\pi_{1}=\pi_{2}=1 / 2$, by weighted utility theory, ${ }^{16}$ we have

$f \succcurlyeq g \Leftrightarrow \frac{\frac{1}{2} u(f(1)) w(f(1))+\frac{1}{2} u(f(2)) 2 w(f(2))}{\frac{1}{2} w(f(1))+\frac{1}{2} 2 w(f(2))} \geq \frac{\frac{1}{2} u(g(1)) w(g(1))+\frac{1}{2} u(g(2)) 2 w(g(2))}{\frac{1}{2} w(g(1))+\frac{1}{2} 2 w(g(2))}$.

The MS definition of subjective probabilities will merge the weight coefficients $b(s)$ with the actual probabilities to obtain the probabilities $\mu_{1}=1 / 3$ and $\mu_{2}=2 / 3$. Moreover, the induced lotteries are:

$$
(\mu \otimes f)_{m}=\left[1, \frac{1}{3} ; 1, \frac{2}{3}\right]=[1 ; 1] \text { and }(\mu \otimes g)_{m}=\left[0, \frac{1}{3} ; 2, \frac{2}{3}\right] .
$$

Applying the weighted utility representation, we obtain:

$$
f \succcurlyeq g \Leftrightarrow \frac{\frac{1}{3} u(1) w(1)+\frac{2}{3} u(1) w(1)}{\frac{1}{3} w(1)+\frac{2}{3} w(1)} \geq \frac{\frac{1}{3} u(0) w(0)+\frac{2}{3} u(2) w(2)}{\frac{1}{3} w(0)+\frac{2}{3} w(2)}=\frac{4}{5} .
$$

Thus the MS definition of subjective probabilities is consistent with the decision-maker's choice behavior but it is at variance with his beliefs.

${ }^{16}$ See Chew (1983). 
Next we show that, in this case, the MS definition of subjective probabilities violates the quantifiable beliefs model. Suppose, by way of negation, that the MS definition of subjective probabilities is consistent with the definition of probabilities according to the theory of quantifiable beliefs. Observe that, given the MS subjective probabilities, the model of quantifiable beliefs requires that $f \succcurlyeq g$ implies $\mu \otimes f \succcurlyeq \mu \otimes g$. But the weighted utility representation implies,

$$
\frac{\frac{1}{3} u(1) w(1)+\frac{2}{3} 2 u(1) w(1)}{\frac{1}{3} w(1)+\frac{2}{3} 2 w(1)} \leq \frac{\frac{1}{3} u(0) w(0)+\frac{2}{3} u(2) 2 w(2)}{\frac{1}{3} w(0)+\frac{2}{3} 2 w(2)}=\frac{8}{7} .
$$

A contradiction.

\subsection{Recursive preferences}

As stated in the introduction, the framework of interim-state lotteries can accommodate recursivity in the decision-making process. The idea is the following: if a decision maker who, were he sure that a particular state $s$ were going to obtain, would strictly prefer to have the roulette lottery $p$ in that state to the roulette lottery $q$, then he should prefer any act (respectively, any interim-state lottery) that yields the roulette lottery $p$ in state $s$ over another act (respectively, interim-state lottery) that is identical to the first act (respectively, interim-state lottery) everywhere outside of state $s$, but yields the roulette lottery $q$ in state $s$. For the particular case in which $p>_{s}^{1} q$, this form of recursivity is already implied by intra-state monotonicity, ordinal act independence and transitivity (see Lemma 1). For recursive preferences we extend it to hold for any pair of lotteries by strengthening ordinal act independence to hold for non-degenerate as well as degenerate roulette lotteries.

(A.10) (Lottery Ordinal Act Independence) For any obviously nonnull state $s \in S$, any pair of roulette lotteries $p, q \in L_{s}$, any pair of acts $f, g \in H$ and any probability measure $\mu \in M$, such that $\mu_{s}>0: \mu \otimes\left(f^{-s}, p\right) \succcurlyeq \mu \otimes\left(f^{-s}, q\right)$ if and only if $\left(g^{-s}, p\right) \succcurlyeq\left(g^{-s}, q\right)$.

If a function defined over the set of interim-state lotteries is to induce a preference relation that satisfies (A.10) then it must be a member of the following class of functions.

Definition 10 A function $V: \hat{L} \rightarrow \mathbb{R}$ is said to be recursive if for any act $f \in H$, any state $s \in S$, any probability measure $\mu \in M$, with $\mu_{s}>0$, and any pair of roulette lotteries $p, q \in L_{s}$,

$$
V\left(\delta^{s} \otimes\left((\underline{x})^{-s}, p\right)\right) \geq V\left(\delta^{s} \otimes\left((\underline{x})^{-s}, q\right)\right) \Leftrightarrow V\left(\mu \otimes\left(f^{-s}, p\right)\right) \geq V\left(\mu \otimes\left(f^{-s}, q\right)\right) .
$$


The following corollary to our main representation Theorem 1 states that strengthening (A.4) to (A.10) provides a characterization of preferences that can be represented by quantifiable beliefs and a recursive preference functional.

Corollary 2 Let $\succcurlyeq$ and $\hat{\succcurlyeq}$ be binary relations on $H$ and $\hat{L}$, respectively. Given $\succcurlyeq$ and $\hat{\succcurlyeq}$ satisfy state-preference overlap then the following conditions are equivalent:

(i) The binary relations $\succcurlyeq$ and $\hat{\succcurlyeq}$ satisfy, weak-order, Archimedean, intra-state monotonicity, lottery ordinal act independence, inter-state monotonicity and concordance.

(ii) There exists a unique probability measure $\pi$ on $S$ such that $\pi_{s}=0$ if and only if $s$ is obviously null and a mixture continuous, strictly monotonic and recursive function $V: \hat{L} \rightarrow \mathbb{R}$ such that:

(a) $V$ represents $\hat{\succcurlyeq}$.

(b) For all $f, g \in H$,

$$
f \succcurlyeq g \Leftrightarrow V(\pi \otimes f) \geq V(\pi \otimes g)
$$

In the next subsection we provide an example of a recursive function that generates a preference relation over interim-state lotteries that is consistent with a preference relation over acts that in turn can rationalize behavior consistent with the well-known Ellsberg paradox.

\subsection{Recursivity without Reduction and the Ellsberg Paradox}

Ellsberg (1961) provided the following thought experiment to support his thesis that subjective beliefs need not always be 'quantifiable' by a probability measure. An urn contains 90 balls, of which 30 are known to be red. The other 60 are either black or white, but the decision maker does not know precisely the numbers of each. One ball will be drawn from this urn. Consider the following four "bets".

$\mathbf{B}_{1}$ Win if the ball drawn is red, otherwise lose.

$\mathbf{B}_{2}$ Win if the ball drawn is black, otherwise lose.

$\mathbf{B}_{1}^{\prime}$ Win if the ball drawn is red or white, otherwise lose.

$\mathbf{B}_{2}^{\prime}$ Win if the ball drawn is black or white, otherwise lose. 
Ellsberg predicted that most people would prefer the bet $\mathbf{B}_{1}$ to the bet $\mathbf{B}_{2}$, but would also prefer the bet $\mathbf{B}_{2}^{\prime}$ to the bet $\mathbf{B}_{1}^{\prime}$. Although Ellsberg did not run any formal experiments himself, a number of papers report that his predictions have been borne out. ${ }^{17}$

A number of researchers, including, among others, Gardenfors and Sahlin (1982, 1983) and Segal $(1987,1990)$ have argued that this problem of decision making under uncertainty is best represented as a two-stage process. In the first stage, an urn with an unknown distribution of balls is selected and in the second stage a ball is drawn from this urn. Thus given the information above, the state-space can be taken to consist of sixty-one elements: one for each possible distribution of balls that the urn could conceivably have. That is, for each $s \in\{0,1, \ldots, 60\}, s \in S$ is the state that corresponds to the urn containing 30 red balls, $s$ black balls and $60-s$ white balls. ${ }^{18}$ The set of (state-independent) outcomes is simply the set $X:=\{$ win, lose $\}$. In this framework, the bets $\mathbf{B}_{1}$ and $\mathbf{B}_{2}^{\prime}$ correspond to the 'constant' acts, $f$ and $g^{\prime}$ in which $f_{s}($ win $)=1 / 3$ and $g_{s}^{\prime}($ win $)=2 / 3$, for every $s \in S$. The bets $\mathbf{B}_{2}$ and $\mathbf{B}_{1}^{\prime}$ on the other hand correspond to the non-constant acts, $g$ and $f^{\prime}$ in which $g_{s}$ (win) $=s / 90$ and $f_{s}^{\prime}($ win $)=(90-s) / 90$ for each $s \in S$.

Ellsberg's predicted choices for the two problems are inconsistent with a pair of preference relations over acts and interim-state lotteries that satisfy our theorem and also respect the reduction property embodied in the MS state-independence axiom (A.9). To see this, notice that if $\mu$ were the probability distribution over $S$ that "quantified" the decision-maker's beliefs, then $f \succ g$ would entail $\mu \otimes f \hat{\succ} \mu \otimes g$ and by (A.9) we would have "not $(\mu \otimes g)_{m} \geq_{X}^{1}$ $(\mu \otimes f)_{m} "$, or equivalently,

$$
\frac{1}{3}>\sum_{s=0}^{60} \mu_{s} \frac{s}{90} .
$$

However, $g^{\prime} \succ f^{\prime}$ would require $\mu \otimes g^{\prime} \hat{\succ} \mu \otimes f^{\prime}$ and (A.9) in this case would imply

$$
\frac{2}{3}>\sum_{s=0}^{60} \mu_{s}\left(\frac{90-s}{90}\right) \Leftrightarrow \sum_{s=0}^{60} \mu_{s} \frac{s}{90}>\frac{1}{3},
$$

\footnotetext{
17 See Camerer (1995, p. 646) for references and discussion of the literature on experimental tests of 'ambiguity aversion'.

${ }^{18}$ Given the almost universal acclamation that 2000 is the first year of the new century and of the new millenium, we have acceded to popular will and designated $s=0$ as the first of our sixty-one state representation of the subjective uncertainty embodied in this Ellsberg thought experiment. As a further aside, we suggest that the new century should be designated the twentieth century, thereby allowing us to refer to the 1900s as the nineteenth century, the 1800 s as the eighteenth century, et cetera. Any confusion that this might cause from earlier works of previous centuries that labeled centuries by the archaic practice of thinking of xx00s as the $\mathrm{xx}+1$ century, we believe is worth bearing given the elegant symmetry of being able to refer to the events of the 1400 s as having occurred during the fourteenth century.
} 
a contradiction.

But as Segal (1987) demonstrates (by means of an example) a preference relation over twostage lotteries that does not obey the reduction property embodied in (A.9) can rationalize choices consistent with Ellsberg's prediction.

For example, suppose the preference relation $\hat{\succcurlyeq}$ over the set of interim-state lotteries $\hat{L}$ can be represented by the rank-dependent utility functional

$$
V(\nu \otimes h)=\sum_{i=0}^{59} g\left(\sum_{j=0}^{i} \nu_{\varphi^{-1}(j ; h)}\right)\left[\Pi(h)_{i}(\operatorname{win})-\Pi(h)_{i+1}(\operatorname{win})\right]+\Pi(h)_{60}(\text { win }),
$$

where the probability transformation function, $g$, is given by:

$$
g(p)=\left\{\begin{array}{cc}
p / 2 & \text { if } p \in[0,1 / 2] \\
3 p / 2-1 / 2 & \text { if } p \in(1 / 2,1]
\end{array},\right.
$$

and $\Pi: H \rightarrow H$ is defined by $\Pi(h)_{i}=h_{\varphi(i ; h)}$, where $\varphi(\cdot ; h): S \rightarrow S$ is a permutation satisfying $h_{\varphi(i ; h)}($ win $) \geq h_{\varphi(i+1 ; h)}($ win) for $i=0, \ldots, 59$.

Further suppose, that $\succcurlyeq$ can be represented by the restriction of $\hat{\succcurlyeq}$ to interim-state lotteries whose marginal distribution over the states is the uniform distribution. That is, $\pi_{s}=1 / 61$ for all $s \in S$. Hence, for every pair $h, h^{\prime} \in H, h \succcurlyeq h^{\prime}$ if and only if $V(\pi \otimes h)$ $\geq V\left(\pi \otimes h^{\prime}\right)$. Notice that $\hat{\succcurlyeq}$ and $\succcurlyeq$ conform to the special case characterized in Corollary 2 which can be represented by quantifiable beliefs and a recursive preference functional. Moreover, we have

$$
\begin{aligned}
V(\pi \otimes f) & =\frac{1}{3}>V(\pi \otimes g) \approx 0.238 \\
V\left(\pi \otimes g^{\prime}\right) & =\frac{2}{3}>V\left(\pi \otimes f^{\prime}\right) \approx 0.571
\end{aligned}
$$

An alternative approach to resolving the Ellsberg paradox is presented in Schmeidler (1989). He also adopts the Anscombe-Aumann framework of two-stage horse/roulette lottery acts. As we have argued above, this makes intuitive sense, as the first-stage of subjective uncertainty may be viewed as modeling the lack of information the decision maker has about the precise distribution of balls in the urn. Schmeidler maintains all the axioms of Anscombe and Aumann (1961) except for their independence axiom which he requires only to hold for those acts that are 'pairwise co-monotonic'. That is, for any triple of acts $f, g$ and $h$, if for every pair of states $s$ and $t$, (i) $f_{s} \succ f_{t}$ implies $g_{s} \succcurlyeq g_{t}$ and $h_{s} \succcurlyeq h_{t}$; and (ii) $g_{s} \succ g_{t}$ implies $h_{s} \succcurlyeq h_{t}$; then $f \succcurlyeq g$ implies $\alpha f+(1-\alpha) h \succcurlyeq \alpha g+(1-\alpha) h$, for all $\alpha$ in $(0,1)$. The main 
result of his paper is that these axioms are necessary and sufficient for such a preference relation to admit a Choquet Expected Utility (CEU) representation of the form

$$
C E U(h)=\sum_{i=0}^{59} \psi\left(\left\{\varphi^{-1}(0 ; h), \ldots, \varphi^{-1}(i ; h)\right\}\right)\left[u\left(\Pi(h)_{i}\right)-u\left(\Pi(h)_{i+1}\right)\right]+u\left(\Pi(h)_{60}\right),
$$

where $\psi: 2^{S} \rightarrow[0,1]$, with $\psi(\emptyset)=0, \psi(S)=1$ and for all $A, B \subset S, A \subset B \Rightarrow \psi(A) \leq$ $\psi(B)$.

A (normalized) capacity $\psi$ can be viewed as a generalization of a probability measure that need not satisfy the property of additivity (that is, $\mu(A \cup B)=\mu(A)+\mu(B)-\mu(A \cap B)$ ). Indeed, Schmeidler argues that the concept of 'uncertainty aversion' that is offered as a possible explanation for the Ellsberg paradox can be formally captured in the CEU model in the Anscombe-Aumann framework with a capacity that is convex, namely, $\psi(A \cup B) \geq$ $\psi(A)+\psi(B)-\psi(A \cap B)$ (with strict inequality for pairs of 'ambiguous events').

Notice, however, that the functional defined in (3) may also be viewed as a member of the class of CEU functionals. The capacity $\psi$ is given by $\psi(E)=g\left(\sum_{s \in E} \pi_{s}\right)$ for each $E \subset S$, and since $g(\cdot)$ is convex, it follows that $\psi$ is a convex capacity. The (expected) utility function is given by $u\left(h_{s}\right)=h_{s}$ (win) for every $h \in H$ and every $s \in S$. Thus, in addition to reinforcing the insight of Segal (1987) that Ellsberg-type behavior can be accommodated in a two-stage lottery framework in which reduction is dropped but recursivity is retained, the example above also suggests that once we dispense with reduction we may still have choice behavior under uncertainty that accords with the predictions of the so-called non-additive belief models of Choquet Expected Utility theory or Maxmin Expected Utility but which may yet allow for beliefs that are quantifiable with a probability measure in the sense of the Theorem 1. 


\section{APPENDIX}

A. Proof of Lemma 1: Fix an obviously nonnull state $s$, and any act $f$. The lemma follows immediately from the following well-known fact: if $p$ first order stochastically dominates $p^{\prime}$ then there exists a finite sequence of roulette lotteries $\left(p_{k}\right)_{k=1}^{N}$, for which $p_{1}=p$, $p_{N}=p^{\prime}$ and for every $k=1, \ldots, N-1$, there exists $q_{k} \in L_{s}, x_{k}, y_{k} \in X_{s}$ and $\alpha_{k} \in[0,1]$, satisfying, $x_{k} \hat{\succcurlyeq}_{s} y_{k}, p_{k}=\left(1-\alpha_{k}\right) q_{k}+\alpha_{k} \delta^{y_{k}}$ and $p_{k+1}=\left(1-\alpha_{k}\right) q_{k}+\alpha_{k} \delta^{x_{k}}$. Hence, by intra-state monotonicity and ordinal act independence, we have $\left(f^{-s}, p_{k}\right) \succcurlyeq\left(f^{-s}, p_{k+1}\right)$ and $\mu \otimes\left(f^{-s}, p_{k}\right) \succcurlyeq \mu \otimes\left(f^{-s}, p_{k+1}\right)$ for all $k=1, \ldots, N-1$. The conclusion follows by transitivity.

B. Proof of Lemma 2: Since $\bar{x} \succ \underline{x}$, by ordinal act independence it follows that there is at least one obviously nonnull state. Partition the set of states into two subsets, $E$ and $N$, where $E$ is the set of obviously nonnull states and $N$ is the set of obviously null states. By state-preference overlap it follows that there exists a state $s \in E$ such that

$$
\delta^{t} \otimes \bar{x} \succcurlyeq \delta^{s} \otimes \bar{x} \hat{\succ} \delta^{t} \otimes \underline{x} \succcurlyeq \delta^{s} \otimes \underline{x} \text { for all } t \in E .
$$

Ordinal act independence and state-preference overlap also imply that for any $\mu \in M$, with $\mu_{s}, \mu_{t}>0$,

$$
\mu \otimes\left(\left(\underline{x}^{-s}, \bar{x}_{s}\right)^{-t}, \bar{x}_{t}\right) \hat{\succ} \mu \otimes\left(\underline{x}^{-s}, \bar{x}_{s}\right) \hat{\succ} \mu \otimes \underline{x} .
$$

Furthermore, ordinal act independence also implies

$$
\left(\left(\underline{x}^{-s}, \bar{x}_{s}\right)^{-t}, \bar{x}_{t}\right) \succ\left(\underline{x}^{-s}, \bar{x}_{s}\right) \succ \underline{x}
$$

for every obviously nonnull state $t \in E$; and

$$
\left(\left(\underline{x}^{-s}, \bar{x}_{s}\right)^{-t}, \bar{x}_{t}\right) \sim\left(\underline{x}^{-s}, \bar{x}_{s}\right) \succ \underline{x}
$$

for every obviously null state $t \in N$.

By mixture solvability of $\succcurlyeq$, there exists a unique set of $(n-1)$ numbers $\left(\gamma_{t}\right)_{t \in S-\{s\}}$ that satisfy, $\gamma_{t} \in(0,1]$ and

$$
\left(\underline{x}^{-s}, \bar{x}_{s}\right) \sim \gamma_{t}\left(\left(\underline{x}^{-s}, \bar{x}_{s}\right)^{-t}, \bar{x}_{t}\right)+\left(1-\gamma_{t}\right) \underline{x} .
$$


Notice that for any obviously null state $t \in N, \gamma_{t}=1$.

Similarly, by mixture solvability of $\hat{\succcurlyeq}$, it follows that for each $\mu \in M$, with $\mu_{s}>0$ there exists a unique set of $(n-1)$ numbers $\left(\hat{\gamma}_{t}\right)_{t \in S-\{s\}}$ such that $\hat{\gamma}_{t} \in(0,1]$ and

$$
\mu \otimes\left(\underline{x}^{-s}, \bar{x}_{s}\right) \hat{\sim} \hat{\gamma}_{t}\left(\mu \otimes\left(\left(\underline{x}^{-s}, \bar{x}_{s}\right)^{-t}, \bar{x}_{t}\right)\right)+\left(1-\hat{\gamma}_{t}\right)(\mu \otimes \underline{x}) .
$$

Our aim is to show that there exist $\mu \in M$ for which $\left(\hat{\gamma}_{t}\right)_{t \in S-\{s\}}$ that satisfy (5) equal $\left(\gamma_{t}\right)_{t \in S-\{s\}}$ that satisfy $(4)$.

First notice that, for any $\mu \in M$ with $\mu_{s}>0, \mu_{t}=0$ if and only if $\hat{\gamma}_{t}=1$. So we shall restrict our attention to probability distributions $\mu \in M$, for which $\mu_{t}>0$ for all $t \in E$ and $\mu_{t}=0$ for all $t \in N$. Let $\bar{M}$ denote this restriction of $M$.

For each $\mu \in \bar{M}$, set $z_{t}(\mu):=\hat{\gamma}_{t}(\mu)-\gamma_{t}$ for each $t \in S-\{s\}$, and set

$$
z_{s}(\mu):=-\frac{\sum_{t \in E-\{s\}} \mu_{t} z_{t}(\mu)}{\mu_{s}} .
$$

Since $z_{t}(\mu)=0$ for every obviously-null state $t \in N$, we have by construction $\sum_{t \in S} \mu_{t} z_{t}(\mu)=$ 0 . Define on $\bar{M}$ the function $z^{+}: \bar{M} \rightarrow \mathbb{R}_{+}^{N}$, by $z_{t}^{+}(\mu)=\max \left\{z_{t}(\mu), 0\right\}$. Continuity of $z^{+}(\cdot)$ (in the topology of $\mathbb{R}^{n}$ ) follows from the mixture continuity of $\hat{\succcurlyeq}$. Denote $\lambda(\mu)=$ $1 /\left(\sum_{t \in E}\left[\mu_{t}+z_{t}^{+}(\mu)\right]\right)$. We have $\lambda(\mu) \in(0,1]$ for all $\mu \in \bar{M}$. Define a continuous function $g: \bar{M} \rightarrow \bar{M}$, by $g(\mu)=\lambda(\mu)\left(\mu+z^{+}(\mu)\right) \cdot{ }^{19} \quad$ By Brouwer's fixed point theorem there is a $\mu^{*} \in \bar{M}$, such that $\mu^{*}=g\left(\mu^{*}\right)$. Furthermore

$$
0=\sum_{t \in S} \mu_{t}^{*} z_{t}\left(\mu^{*}\right)=\sum_{t \in S} g_{t}\left(\mu^{*}\right) z_{t}\left(\mu^{*}\right)=\lambda\left(\mu^{*}\right) \sum_{t \in S} z_{t}^{+}\left(\mu^{*}\right) z_{t}\left(\mu^{*}\right) .
$$

Notice that for any $t$, if $z_{t}\left(\mu^{*}\right)<0$ then $z_{t}\left(\mu^{*}\right) z_{t}^{+}\left(\mu^{*}\right)=0$. Hence (6) implies

$$
z_{t}\left(\mu^{*}\right) \leq 0 \text { for all } t \in S \text {. }
$$

Since $z_{t}\left(\mu^{*}\right)=0$ for all $t \in N$, if $(7)$ did not hold with equality for all $t$, then for some $t^{\prime} \in E$, we would have $z_{t^{\prime}}<0$. But since $\gamma_{t^{\prime}} \in(0,1)$, it follows from $(5)$ that $\mu_{\hat{t}}^{*}>0$, contradicting (6). Hence we have $z_{t}\left(\mu^{*}\right)=0$ for all $t \in S$, that is, for $\mu^{*}$ we have

$$
\mu^{*} \otimes\left(\underline{x}^{-s}, \bar{x}_{s}\right) \hat{\sim} \gamma_{t}\left(\mu^{*} \otimes\left(\left(\underline{x}^{-s}, \bar{x}_{s}\right)^{-t}, \bar{x}_{t}\right)\right)+\left(1-\gamma_{t}\right)\left(\mu^{*} \otimes \underline{x}\right)
$$

\footnotetext{
19 The construction of the function $g$, a continuous mapping of the simplex $\bar{M}$ into itself draws heavily on the section in Mas-Colell, Whinston \& Green (1995, pp. 586-7) that provides a proof of the existence of a general equilibrium in an exchange economy for which aggregate excess demand is a 'well-behaved' function of prices.
} 
$>$ From the construction of $\mu^{*}$ in the previous step, the definition of the mixture operation and mixture monotonicity, we have for any $t \in S-\{s\}$,

$\mu^{*} \otimes\left[\begin{array}{ccc}\bar{x}_{s} & \text { on } & s \\ \underline{x}_{t} & \text { on } & t \\ \underline{x}_{s^{\prime}} & \text { on } & s^{\prime} \in S-\{s, t\}\end{array}\right] \succcurlyeq \mu^{*} \otimes\left[\begin{array}{ccc}\alpha \delta^{\bar{x}_{s}}+(1-\alpha) \delta \underline{x}_{s} & \text { on } & s \\ \alpha \delta^{\bar{x}_{t}}+(1-\alpha) \delta \underline{x}_{t} & \text { on } & t \\ \underline{x}_{s^{\prime}} & \text { on } & s^{\prime} \in S-\{s, t\}\end{array}\right] \Leftrightarrow \gamma_{t} \geq \alpha$.

And (4) and mixture monotonicity imply

$$
\left[\begin{array}{ccc}
\bar{x}_{s} & \text { on } & s \\
\underline{x}_{t} & \text { on } & t \\
\underline{x}_{s^{\prime}} & \text { on } & s^{\prime} \in S-\{s, t\}
\end{array}\right] \succcurlyeq\left[\begin{array}{ccc}
\alpha \delta^{\bar{x}_{s}}+(1-\alpha) \delta \underline{x}_{s} & \text { on } & s \\
\alpha \delta^{\bar{x}_{t}}+(1-\alpha) \delta \underline{x}_{t} & \text { on } & t \\
\underline{x}_{s^{\prime}} & \text { on } & s^{\prime} \in S-\{s, t\}
\end{array}\right] \Leftrightarrow \gamma_{t} \geq \alpha .
$$

Thus, by definition of $\alpha_{t}(\mu)$ and $\beta_{t}$, for all $t \in S-\{s\}, \beta_{t}=\alpha_{t}\left(\mu^{*}\right)$.

C. Proof of Lemma 3: Since $p \geq_{s}^{1}\left(>_{s}^{1}\right) p^{\prime}$ implies $\alpha p+(1-\alpha) p^{\prime} \geq_{s}^{1}\left(>_{s}^{1}\right) \beta p+(1-\beta) p^{\prime}$ for all $\alpha, \beta \in[0,1]$ such that $\alpha \geq \beta(\alpha>\beta)$, the conclusion follows for $\succcurlyeq$ directly from state by state repeated applications of Lemma 1 . The result for $\hat{\succcurlyeq}$ follows by the same reasoning using inter-state monotonicity and the fact that $\geq_{\hat{L}}$ is the transitive closure of $>_{\hat{L}}^{1}$.

D. Proof of Lemma 5: (By induction). From Lemmas 3 and 4, it follows that $\succcurlyeq$ and $\hat{\succcurlyeq}$ satisfy mixture solvability. By concordance mixture monotonicity and mixture solvability the lemma holds for any two-element event $\{s, t\}, t \in S-\{s\}$. So assume that it holds for any event $E$ that consists of no more than $k$ states and of which the state $s$ is an element. By the induction assumption there exists a unique $\beta_{1} \in[0,1]$ such that

$$
\mu \otimes f \hat{\sim} \mu \otimes\left[\begin{array}{ccc}
\beta_{1} g_{t}+\left(1-\beta_{1}\right) h_{t} & \text { on } & t \in E-\{s\} \\
\beta_{1} g_{s}+\left(1-\beta_{1}\right) h_{s} & \text { on } & s \\
f_{\widehat{s}} & \text { on } & \widehat{s} \\
f_{s^{\prime}} & \text { on } & s^{\prime} \notin E \cup\{\widehat{s}\}
\end{array}\right]
$$


if and only if

$$
f \sim\left[\begin{array}{ccc}
\beta_{1} g_{t}+\left(1-\beta_{1}\right) h_{t} & \text { on } & t \in E-\{s\} \\
\beta_{1} g_{s}+\left(1-\beta_{1}\right) h_{s} & \text { on } & s \\
f_{\widehat{s}} & \text { on } & \widehat{s} \\
f_{s^{\prime}} & \text { on } & s^{\prime} \notin E \cup\{\widehat{s}\}
\end{array}\right] .
$$

By transitivity (of indifference), mixture solvability and concordance there exists a unique $\beta_{2} \in[0,1]$ such that

$$
\mu \otimes f \hat{\sim} \mu \otimes\left[\begin{array}{ccc}
\beta_{1} g_{t}+\left(1-\beta_{1}\right) h_{t} & \text { on } & t \in E-\{s\} \\
\beta_{2} g_{s}+\left(1-\beta_{2}\right) h_{s} & \text { on } & s \\
\beta_{2} g_{\widehat{s}}+\left(1-\beta_{2}\right) h_{\widehat{s}} & \text { on } & \widehat{s} \\
f\left(s^{\prime}\right) & \text { on } & s^{\prime} \notin E \cup\{\widehat{s}\}
\end{array}\right]
$$

if and only if

$$
f \sim\left[\begin{array}{ccc}
\beta_{1} g_{t}+\left(1-\beta_{1}\right) h_{t} & \text { on } & t \in E-\{s\} \\
\beta_{2} g_{s}+\left(1-\beta_{2}\right) h_{s} & \text { on } & s \\
\beta_{2} g_{\widehat{s}}+\left(1-\beta_{2}\right) h_{\widehat{s}} & \text { on } & \widehat{s} \\
f\left(s^{\prime}\right) & \text { on } & s^{\prime} \notin E \cup\{\widehat{s}\}
\end{array}\right] .
$$

If $\beta_{1}=\beta_{2}$ then we are done. Otherwise suppose, without loss of generality, that $\beta_{2}>\beta_{1}$. By transitivity (of indifference), the induction assumption and mixture solvability there exists $\beta_{3} \in[0,1]$ such that

$$
\mu \otimes f \hat{\sim} \mu \otimes\left[\begin{array}{ccc}
\beta_{3} g_{t}+\left(1-\beta_{3}\right) h_{t} & \text { on } & t \in E-\{s\} \\
\beta_{3} g_{s}+\left(1-\beta_{3}\right) h_{s} & \text { on } & s \\
\beta_{2} g_{\widehat{s}}+\left(1-\beta_{2}\right) h_{\widehat{s}} & \text { on } & \widehat{s} \\
f\left(s^{\prime}\right) & \text { on } & s^{\prime} \notin E \cup\{\widehat{s}\}
\end{array}\right]
$$


if and only if

$$
f \sim\left[\begin{array}{ccc}
\beta_{3} g_{t}+\left(1-\beta_{3}\right) h_{t} & \text { on } & t \in E-\{s\} \\
\beta_{3} g_{s}+\left(1-\beta_{3}\right) h_{s} & \text { on } & s \\
\beta_{2} g_{\widehat{s}}+\left(1-\beta_{2}\right) h_{\widehat{s}} & \text { on } & \widehat{s} \\
f\left(s^{\prime}\right) & \text { on } & s^{\prime} \notin E \cup\{\widehat{s}\}
\end{array}\right]
$$

Mixture monotonicity implies $\beta_{3} \in\left(\beta_{1}, \beta_{2}\right)$. Reiterating this procedure, we produce two sequences: $\beta_{1}<\beta_{3}<\ldots<\beta_{2 i+1}<\ldots$ and $\beta_{2}>\beta_{4}>\ldots>\beta_{2 i}>\ldots$ Both sequences are bounded above by 1 and below by 0 , and so the first sequence has a least upper bound, $\bar{b}$, and the second sequence has a greatest lower bound, $\underline{b}$. Furthermore, by mixture solvability, there is a unique $\beta \in(0,1)$ such that

$$
f \sim\left[\begin{array}{ccc}
\beta g_{t}+(1-\beta) h_{t} & \text { on } & t \in E-\{s\} \\
\beta g_{s}+(1-\beta) h_{s} & \text { on } & s \\
\beta g_{\widehat{s}}+(1-\beta) h_{\widehat{s}} & \text { on } & \widehat{s} \\
f\left(s^{\prime}\right) & \text { on } & s^{\prime} \notin E \cup\{\widehat{s}\}
\end{array}\right] .
$$

Transitivity and the definition of $\bar{b}$ and $\underline{b}$ imply $\beta=\bar{b}=\underline{b}$. This completes the proof.

E. Proof of Theorem 1: $(i) \Rightarrow(i i)$. Deriving a representation of $\hat{\succcurlyeq}^{20}$ For any $(s, y),(t, z) \in C$, such that $\delta^{t} \otimes\left(\bar{x}^{-t}, z\right) \hat{\succ} \delta^{s} \otimes\left(\underline{x}^{-s}, y\right)$ define the (open) "order-interval"

$$
I((s, y),(t, z))=\left\{\mu \otimes f \in \hat{L}: \delta^{t} \otimes\left(\bar{x}^{-t}, z\right) \hat{\succ} \mu \otimes f \hat{\succ} \delta^{s} \otimes\left(\underline{x}^{-s}, y\right)\right\} .
$$

Fix $s \in S$. For each $\mu \otimes f \in I\left(\left(s, \underline{x}_{s}\right),\left(s, \bar{x}_{s}\right)\right)$, by mixture solvability there exists a unique number $\alpha(\mu \otimes f) \in(0,1)$ such that

$$
\mu \otimes f \hat{\sim} \alpha(\mu \otimes f)\left(\delta^{s} \otimes \bar{x}\right)+(1-\alpha(\mu \otimes f))\left(\delta^{s} \otimes \underline{x}\right)
$$

Moreover, for any other $\nu \otimes g \in I\left(\left(s, \underline{x}_{s}\right),\left(s, \bar{x}_{s}\right)\right)$, by mixture monotonicity

$$
\alpha(\mu \otimes f) \geq \alpha(\nu \otimes g) \Leftrightarrow \mu \otimes f \hat{\succcurlyeq} \nu \otimes g
$$

\footnotetext{
${ }^{20}$ The following argument draws heavily on an analogous result by Grant and Atsushi Kajii which appeared in Grant (1995, Proof of Theorem II, Step 5, pp. 187-188).
} 
Define $F: I\left(\left(s, \underline{x}_{s}\right),\left(s, \bar{x}_{s}\right)\right) \rightarrow(1 / 4,3 / 4)$ by $F(\mu \otimes f)=\alpha(\mu \otimes f) / 2+1 / 4$.

The method of proof is to show that the set $\left\{\mu \otimes f \in \hat{L}: \mu \otimes f \hat{\succ} \delta^{s} \otimes \bar{x}\right\}$ can be covered by a countable union of nested "order-intervals" and then to show that $\hat{\succcurlyeq}$ restricted to this set can be represented by a function whose range is a subset of $(3 / 4,1]$. A similar argument can then be applied to the set $\left\{\mu \otimes f \in \hat{L}: \delta^{s} \otimes \underline{x} \hat{\succ} \mu \otimes f\right\}$ to demonstrate that $\hat{\succcurlyeq}$ restricted to that set can be represented by a function whose range is a subset of $[0,1 / 4)$.

Consider the set $U$ that consists of pairs of the form $\left(I^{U}, F^{U}\right)$ where $I^{U}$ is the countable union of the set of intervals $\left\{I\left(\left(s, \bar{x}_{s}\right),\left(t^{1}, z^{1}\right)\right), I\left(\left(s, \bar{x}_{s}\right),\left(t^{2}, z^{2}\right)\right), \ldots\right\}$ with $\left(t^{i}, z^{i}\right) \in C$ and $\delta^{t^{i+1}} \otimes\left(\bar{x}^{-t^{i+1}}, z^{i+1}\right) \hat{\succ} \delta^{t^{i}} \otimes\left(\bar{x}^{-t^{i}}, z^{i}\right)$ for all $i=1,2, \ldots ;$ and $F^{U}: \hat{L} \rightarrow[3 / 4,1]$ that represents $\hat{\succcurlyeq}$ on $I^{U}$. For every $\mu \otimes f \in I\left(\delta^{t^{i-1}} \otimes\left(\bar{x}^{-t^{i-1}}, z^{i-1}\right), \delta^{t^{i}} \otimes\left(\bar{x}^{-t^{i}}, z^{i}\right)\right)$, where $t^{0}:=s$, define $\lambda_{i}(\mu \otimes f)$ by

$$
\mu \otimes f \hat{\sim} \lambda_{i}(\mu \otimes f)\left(\delta^{t^{i}} \otimes\left(\bar{x}^{-t^{i}}, z^{i}\right)\right)+\left(1-\lambda_{i}(\mu \otimes f)\right)\left(\delta^{t^{i-1}} \otimes\left(\bar{x}^{-t^{i-1}}, z^{i-1}\right)\right) .
$$

We can find an appropriate $F^{U}$ by taking the limit of a sequence of functions $F_{i}^{U}$ defined as:

$$
\begin{aligned}
& F_{1}^{U}(\mu \otimes f)=\left\{\begin{array}{cccc}
3 / 4 & \text { if } & \delta^{s} \otimes \bar{x} \succcurlyeq \mu \otimes f \\
3 / 4+\lambda_{1}(\mu \otimes f) / 8 & \text { if } & \mu \otimes f \in I\left(\delta^{s} \otimes \bar{x}, \delta^{1} \otimes\left(\bar{x}^{-t^{1}}, z^{1}\right)\right) \\
7 / 8 & \text { if } & \mu \otimes f \hat{\succcurlyeq} \delta^{t^{1}} \otimes\left(\bar{x}^{-t^{1}}, z^{1}\right)
\end{array}\right.
\end{aligned}
$$

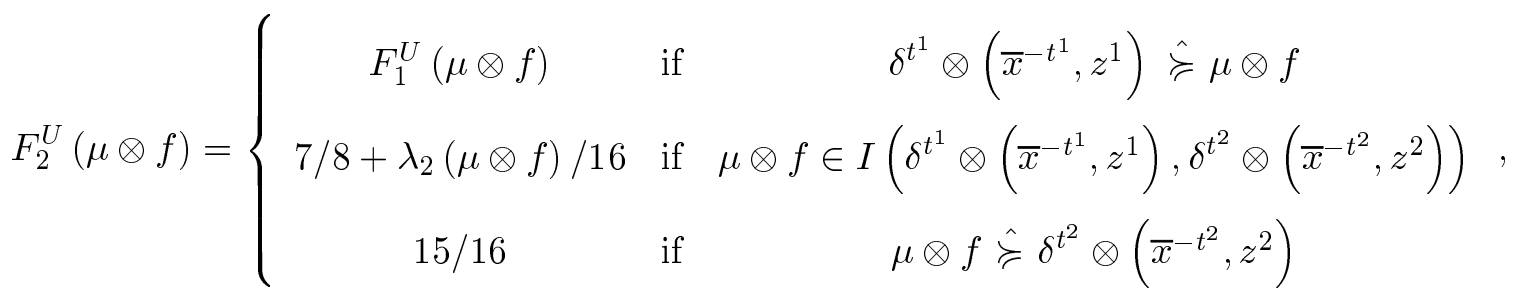

$$
\begin{aligned}
& F_{i}^{U}(\mu \otimes f)=\left\{\begin{array}{ccc}
F_{i-1}^{U}(\mu \otimes f) & \text { if } & \delta^{t^{i-1}} \otimes\left(\bar{x}^{-t^{i-1}}, z^{i-1}\right) \hat{\succcurlyeq} \mu \otimes f \\
1-2^{-i-1}+2^{-i-2} \times \lambda_{i}(\mu \otimes f) & \text { if } & \mu \otimes f \in I\left(\delta^{t^{i-1}} \otimes\left(\bar{x}^{-t^{i-1}}, z^{i-1}\right), \delta^{t^{i}} \otimes\left(\bar{x}^{-t^{i}}, z^{i}\right)\right) \\
1-2^{-i-2} & \text { if } & \mu \otimes f \hat{f} \delta^{t^{i}} \otimes\left(\bar{x}^{-t^{i}}, z^{i}\right)
\end{array}\right.
\end{aligned}
$$


$U$ can be endowed with the following ordering, $\geq_{U}$, defined as follows: $\left(I^{U}, F^{U}\right) \geq_{U}\left(I^{U^{\prime}}, F^{U^{\prime}}\right)$ if and only if $I^{U^{\prime}} \subseteq I^{U}$. Clearly, $\geq_{U}$ is a partial order. Thus, by the Hausdorff Maximal Principle (see Royden (1988, p.25)), there exists a maximal linearly ordered (with respect to $\geq_{U}$ ) set $U^{*} \subset U$. Let $I^{U^{*}}$ be the union of $I^{U}$ 's in $U^{*}$. Define $F^{U^{*}}$ naturally, that is, $F^{U^{*}}(\mu \otimes f)=F^{U}(\mu \otimes f)$ where $F^{U}$ is such that $\left(I^{U}, F^{U}\right)$ is in $U^{*}$ and $\mu \otimes f \in I^{U^{*}}$. Notice that although there may be many $F^{U}$ 's that satisfy the condition above, $F^{U^{*}}$ is well defined since $F^{U}(\mu \otimes f)=F^{U^{\prime}}(\mu \otimes f)$ for all $F^{U}$ and $F^{U^{\prime}}$ such that $\left(I^{U}, F^{U}\right)$ and $\left(I^{U^{\prime}}, F^{U^{\prime}}\right)$ are in $U^{*}$.

Claim: $I^{U^{*}}=\left\{\mu \otimes f \in \hat{L}: \mu \otimes f \hat{\succ} \delta^{s} \otimes \bar{x}\right\}$.

Proof of Claim: Suppose there is an $\nu \otimes g \in\left\{\mu \otimes f \in \hat{L}: \mu \otimes f \hat{\succ} \delta^{s} \otimes \bar{x}\right\}$ that is not in $I^{U^{*}}$. The fact that for each $s \in S, g_{s}$ is a simple roulette lottery on $X_{s}$ together with inter-state monotonicity, ordinal act independence and intra-state monotonicity imply that there exists $\left(t^{\prime}, z^{\prime}\right) \in C$ such that $\delta^{t^{\prime}} \otimes\left(\bar{x}^{-t^{\prime}}, z^{\prime}\right) \succcurlyeq \nu \otimes g$. Hence the set $U^{*}$ can be extended by adding the interval $I\left(\left(s, \bar{x}_{s}\right),\left(t^{\prime}, z^{\prime}\right)\right)$. That is $U^{*}$ is not maximal, a contradiction. This completes the proof of the Claim.

By analogous reasoning we can define a function $F^{L^{*}}: \hat{L} \rightarrow[0,1 / 4)$ that represents $\hat{\succcurlyeq}$ on the set $\left\{\mu \otimes f \in \hat{L}: \delta^{s} \otimes \underline{x} \hat{\succ} \mu \otimes f\right\}$. So the function

$$
V(\mu \otimes f)=\left\{\begin{array}{cccc}
F^{L^{*}}(\mu \otimes f) & \text { if } & \delta^{s} \otimes \underline{x} \hat{\succ} \mu \otimes f \\
1 / 4 & \text { if } & \mu \otimes f \hat{\sim} \delta^{s} \otimes \underline{x} \\
F(\mu \otimes f) & \text { if } & \mu \otimes f \in I\left(\delta^{s} \otimes \underline{x}, \delta^{s} \otimes \bar{x}\right) \\
3 / 4 & \text { if } & \mu \otimes f \hat{\sim} \delta^{s} \otimes \bar{x} \\
F^{U^{*}}(\mu \otimes f) & \text { if } & \mu \otimes f \hat{\succ} \delta^{s} \otimes \bar{x}
\end{array}\right.
$$

represents $\hat{\succcurlyeq}$.

Existence and uniqueness of $\pi$. Let $\pi=\mu^{*}$. Lemma 5 implies that $V$, restricted to interim-state lotteries of the form $\pi \otimes f, f \in H$, represents $\succsim$. This shows that $\pi$ exists.

To prove the uniqueness of $\pi$ suppose, by way of negation, that there exist $\pi, \pi^{\prime} \in M$ for which (iib) holds and $\pi^{\prime} \neq \pi$. Then, by (iib), for all $f, g \in H$,

$$
f \succcurlyeq g \Leftrightarrow V(\pi \otimes f) \geq V(\pi \otimes g) \Leftrightarrow V\left(\pi^{\prime} \otimes f\right) \geq V\left(\pi^{\prime} \otimes g\right) .
$$


In particular this implies $\pi_{t}^{\prime}=\pi_{t}=0$, for all obviously null states $t \in S$. Set $T:=$ $\left\{t \in S: \pi_{t}^{\prime}>\pi_{t}\right\} . T$ is non-empty and hence contains at least one obviously nonnull state.

State-preference overlap implies that there exists a state $s^{*} \in S$ such that, for all $t \in S$,

$$
\delta^{t} \otimes \bar{x} \succcurlyeq \delta^{s^{*}} \otimes \bar{x} \hat{\succ} \delta^{t} \otimes \underline{x} .
$$

Similarly, state-preference overlap also implies that there exists a state $s_{*} \in S$, such that for all $t \in S$

$$
\delta^{t} \otimes \bar{x} \hat{\succ} \delta^{s_{*}} \otimes \underline{x} \hat{\succcurlyeq} \delta^{t} \otimes \underline{x} .
$$

Mixture monotonicity and mixture solvability imply there exist two acts $g, h \in H$ uniquely defined as follows: for each $t \in S$

$$
\begin{aligned}
& g_{t}=\alpha_{t}\left(\delta^{t} \otimes \bar{x}\right)+\left(1-\alpha_{t}\right)\left(\delta^{t} \otimes \underline{x}\right) \hat{\sim} \delta^{s^{*}} \otimes \bar{x} \\
& h_{t}=\beta_{t}\left(\delta^{t} \otimes \bar{x}\right)+\left(1-\beta_{t}\right)\left(\delta^{t} \otimes \underline{x}\right) \hat{\sim} \delta^{s_{*}} \otimes \underline{x}
\end{aligned}
$$

Notice that, by construction, for each $s \in S, g_{s}>_{s}^{1} h_{s}$. Thus we have

$$
g \succ\left[\begin{array}{ccc}
g_{s} & \text { on } & s \in T \\
h_{t} & \text { on } & t \notin T
\end{array}\right] \succ h .
$$

and (by mixture solvability) a unique $\beta$ for which

$$
\beta g+(1-\beta) h \sim\left[\begin{array}{ccc}
g_{s} & \text { on } & s \in T \\
h_{t} & \text { on } & t \notin T
\end{array}\right] .
$$

Furthermore since (iib) holds for $\pi$ we have

$$
\pi \otimes(\beta g+(1-\beta) h) \hat{\sim} \pi \otimes\left[\begin{array}{lll}
g_{s} & \text { on } & s \in T \\
h_{t} & \text { on } & t \notin T
\end{array}\right] .
$$

Next observe that, for every ordered pair of states $(s, t) \in S \times S,\left(s, g_{s}\right)>_{C}^{1}\left(t, h_{t}\right)$ and $\left(s, \beta g_{s}+(1-\beta) h_{s}\right) \geq_{C}^{1}\left(t, \beta g_{t}+(1-\beta) h_{t}\right)$. Hence, by inter-state monotonicity, if $\pi_{s}^{\prime}>\pi_{s}$ for all $s \in T$, and $\pi_{t}^{\prime} \leq \pi_{t}$ for all $t \notin T$, then

$$
\begin{gathered}
\pi^{\prime} \otimes\left[\begin{array}{ccc}
g_{s} & \text { on } & s \in T \\
h_{t} & \text { on } & t \notin T
\end{array}\right] \hat{\succ} \pi \otimes\left[\begin{array}{ccc}
g_{s} & \text { on } & s \in T \\
h_{t} & \text { on } & t \notin T
\end{array}\right] \\
\hat{\sim} \pi \otimes(\beta g+(1-\beta) h) \hat{\sim} \pi^{\prime} \otimes(\beta g+(1-\beta) h) .
\end{gathered}
$$


But this contradicts (iib) holding for $\pi^{\prime}$.

The proof of $($ ii $) \Rightarrow(i)$ is relatively straightforward and thus omitted.

E. Proof of Lemma 6: (i) $\succcurlyeq$ satisfies (A.3). Fix $s \in S, x, y \in X, p \in L, f \in H$, $\mu \in M$ and $\alpha \in(0,1]$. If $\mu \otimes\left(f^{-s}, x\right) \succcurlyeq(\hat{\succ}) \mu \otimes\left(f^{-s}, y\right)$ then (A.4) implies $x \succcurlyeq(\succ) y$ and so it follows from the definition of $\geq_{X}^{1}\left(>_{X}^{1}\right)$ that $\left(\mu \otimes\left(f^{-s}, x\right)\right)_{m} \geq_{X}^{1}\left(>_{X}^{1}\right)\left(\mu \otimes\left(f^{-s}, y\right)\right)_{m}$. Hence, by (A.9), $\mu \otimes\left(f^{-s}, \alpha \delta^{x}+(1-\alpha) p\right) \succcurlyeq(\hat{\succ}) \mu \otimes\left(f^{-s}, \alpha \delta^{y}+(1-\alpha) p\right)$, as required.

(ii) $\hat{\succcurlyeq} \operatorname{satisfies}\left(\right.$ A.5). Fix $s, t \in S, p, q \in L, f \in H, \mu, \nu \in M$. If $(s, p) \geq_{C}^{1}\left(>_{C}^{1}\right)$ $(t, q), \nu_{s}>\mu_{s}$ and $\nu_{r}=\mu_{r}$ for all $r \in S-\{s, t\}$, then it follows from the definition of $\geq_{X}^{1}\left(>_{X}^{1}\right)$ that $\left(\nu \otimes\left(\left(f^{-s}, p\right)^{-t}, q\right)\right)_{m} \geq_{X}^{1}\left(>_{X}^{1}\right)\left(\mu \otimes\left(\left(f^{-s}, p\right)^{-t}, q\right)\right)_{m}$. Hence, by (A.9), $\nu \otimes\left(\left(f^{-s}, p\right)^{-t}, q\right) \hat{\succcurlyeq}(\hat{\succ}) \mu \otimes\left(\left(f^{-s}, p\right)^{-t}, q\right)$ as required.

F. Proof of Theorem 3: $(i) \Rightarrow(i i)$ The hypothesis of the theorem implies (A.0). Lemma 6 and (i) imply that $\succcurlyeq$ and $\hat{\succcurlyeq}$ satisfy (A.1)-(A.6). We have just proved that with (A.9) these imply the axioms 5 and 6 of Machina and Schmeidler (1995). Observe that (A.1) implies their axiom 1, (i.e., $\succcurlyeq$ is a weak order) and Lemma 4 implies their axiom 2 (i.e., mixture continuity.) Thus, by the theorem of Machina and Schmeidler (1995) there exists a unique probability measure $\pi$ on $S$ and a mixture continuous, strictly monotonic (with respect to first order stochastic dominance) function $V: L \rightarrow \mathbb{R}$ such that for any two acts, $f, g \in H, f \succcurlyeq g \Leftrightarrow V\left(\sum_{s \in S} \pi_{s} f_{s}\right) \geq V\left(\sum_{s \in S} \pi_{s} g_{s}\right)$.

The proof of $(i i) \Rightarrow(i)$ is straightforward and therefore omitted. 


\section{References}

Anscombe, F.J. and R.J. Aumann. 1963. "A Definition of Subjective-Probability." Annals of Mathematical Statistics, 34: 199-205.

Camerer, C. 1995. "Individual Decision Making". In Handbook of Experimental Economics, J. Kagel and A. Roth, editors, Princeton, Princeton University Press: 587-703.

Chew, S. H. 1983. "A generalization of the Quasilinear Mean with Applications to the Measurement of Income Inequality and Decision Theory Resolving the Allais Paradox." Econometrica 51: 1065-1092.

Ellsberg, D. 1961. "Risk, Ambiguity and the Savage Axioms." Quarterly Journal of Economics 75: 643-669.

Epstein, L. 2000. "Are Probabilities Used in Markets?" Journal of Economic Theory 91: 86-90.

Epstein, L. and M. Le-Breton. 1993. "Dynamically Consistent Beliefs Must Be Bayesian." Journal of Economic Theory 61: 1-22.

Fishburn, P. 1970. Utility Theory for Decision Making. New York: John Wiley \& Sons. Fishburn, P. 1973. "A Mixture-Set Axiomatization of Conditional Subjective Expected Utility." Econometrica 41: 1-25.

Gardenfors, P. and N. Sahlin. 1982. "Unreliable Probabilities, Risk Taking, and Decision Making." Synthese, 53: 1077-1086.

Gardenfors, P. and N. Sahlin. 1983. "Decision Making with Unreliable Probabilities," British Journal of Mathematical and Statistical Psychology, 36: 240-251.

Grant, S. 1995. "Subjective Probability without Monotonicity: or How Machina's Mom May also be Probabilistically Sophisticated." Econometrica 63: 159-89.

Karni, E. 1985. Decision Making under Uncertainty: The Case of State-Dependent Preferences. Cambridge, MA: Harvard University Press.

Karni, E. 1996. "Probabilities and Beliefs." Journal of Risk and Uncertainty 13: 249-62. Karni, E. and P. Mongin. 2000. "On the Determination of Subjective Probability by Choices." Management Science 46: 233-248.

Karni, E., and D. Schmeidler. 1981. "An Expected Utility Theory for State-Dependent Preferences." Working Paper 48-80, Foerder Institute for Economic Research, Tel-Aviv University.

Karni, E., D. Schmeidler and K. Vind. 1983. "On State Dependent Preference and Subjective Probabilities." Econometrica, 51: 1021-32.

Machina, M. 1989. "Dynamic Consistency and Non-Expected Utility Models of Choice under Uncertainty." Journal of Economic Literature 27: 1622-68.

Machina, M. and D. Schmeidler. 1992. "A More Robust Definition of Subjective Probability", Econometrica 60: 745-80.

Machina, M. and D. Schmeidler. 1995. "Bayes without Bernoulli: Minimal Conditions for Probabilistically Sophisticated Choice." Journal of Economic Theory 67: 106-28. 
Mas-Colell, A., M. Whinston and J. Green. 1995. Microeconomic Theory. Oxford, Oxford University Press.

Royden, H.L. 1988. Real Analysis (Third Edition). New York: Macmillan.

Savage, L.J. 1954. The Foundations of Statistics. New York: Dover Publications; second revised edition 1972 .

Sarin, R. and P. Wakker. 1992. "A Simple Axiomatization of Nonadditive Expected Utility." Econometrica 60: 1255-72.

Schervish, M.J., T. Seidenfeld and J.B. Kadane. 1990. "State-Dependent Utilities." Journal of the American Mathematical Association 85: 840-7.

Segal, U. 1987. "Ellsberg Paradox and Risk Aversion," International Economic Review 28, 175-201.

Segal, U. 1990. "Two-Stage Lotteries without the Reduction Axiom," Econometrica $58,349-377$.

Schmeidler, D. 1989. "Subjective Probability and Expected Utility without Additivity." Econometrica 57: 571-87.

von Neumann, J. and O. Morgenstern. 1944. Theory of Games and Economic Behavior. Princeton, NJ: Princeton University Press. 